\title{
1 Functional and structural modifications of influenza antibodies during pregnancy
}

3 Madeleine F. Jennewein ${ }^{1 *}$, Martina Kosikova ${ }^{2^{*}}$, Francesca J. Noelette ${ }^{1}$, Peter Radvak ${ }^{2}$, Carolyn M.

4 Boudreau ${ }^{1}$, James D. Campbell ${ }^{3}$, Wilbur H. Chen ${ }^{3}$, Hang Xie ${ }^{2 \#}$, Galit Alter ${ }^{1 \#}$ and Marcela F. Pasetti ${ }^{3,4 \#}$

$6{ }^{1}$ The Ragon Institute of MGH, MIT, and Harvard, Cambridge, MA 02139

$7 \quad{ }^{2}$ Laboratory of Pediatric and Respiratory Viral Diseases, Division of Viral Products, Office of Vaccines

8 Research and Review, Center for Biologics Evaluation and Research, US Food and Drug

$9 \quad$ Administration, Silver Spring, MD, 20993

$10{ }^{3}$ Center for Vaccine Development and Global Health, University of Maryland School of Medicine,

11 Baltimore, MD 21201

$12{ }^{4}$ Lead contact

13

14 * Authors contributed equally to this work

15 \#Corresponding authors: mpasetti@som.umaryland.edu; hang.xie@fda.hhs.gov;

16 galter@mgh.harvard.edu. 


\section{SUMMARY}

19 Pregnancy represents a unique tolerogenic immune state which may alter susceptibility to infection and 20 vaccine-response. Here we characterized humoral immunity to seasonal influenza vaccine strains in

21 pregnant and non-pregnant women. Pregnant women had reduced hemagglutinin subtype-1 (H1)- $\operatorname{lgG}$,

$22 \lg 1$, and $\lg$ 2, hemagglutination inhibition and group 1 and 2 stem $\operatorname{lgG}$. However, H1-specific avidity

23 and FcyR1 binding increased. Influenza-antibodies in pregnancy had distinct Fc and Fab glycans

24 characterized by di-galactosylation and di-sialylation. In contrast, agalactosylation and bisection were

25 prominent outside of pregnancy. H1-specific Fc-functionality was moderately reduced in pregnancy,

26 although likely compensated by stronger binding to cognate antigen and FcR. Multivariate analysis

27 revealed distinct populations characterized by Fc $\gamma \mathrm{R} 1$ binding, H1-IgG levels, and glycosylation. Pooled

28 sera from pregnant women exhibited longer retention in vivo. Our results demonstrate structural and

29 functional modulation of humoral immunity during pregnancy in an antigen-specific manner towards

30 reduced inflammation, increased retention in circulation, and efficient placental transport.

31

32 Keywords: Pregnancy, antibodies, IgG, Fc-functionality, Fc-receptors 
INTRODUCTION

The immune system adapts in a precise and unique way during pregnancy to support fetal development and facilitate full-term delivery. These adaptations involve changes in cell phenotypes and frequencies (Th2 polarization, reduction of circulating NK and T cells, reduction in the number of B cells) as well as modulation of cell function (i.e., cytokine production) (Kraus et al. 2010; Kraus et al. 2012). In addition, antibodies produced during pregnancy display unique glycosylation patterns (Bondt et al. 2014; Bondt et al. 2013; Einarsdottir et al. 2013). Collectively, these changes favor a noninflammatory and tolerogenic environment. As a result, auto-immune conditions, such as rheumatoid arthritis, may transiently resolve during pregnancy (Kraus et al. 2010; Memoli et al. 2013). It has been proposed that a corollary of these alterations is increased maternal susceptibility to pathogens, which would also affect fetal health and development. For example, pregnant women are known to experience more severe influenza virus infection and are at higher risk of death, as evidenced during the 2009 H1N1 pandemic (Kourtis, Read, and Jamieson 2014). During the COVID-19 pandemic, symptomatic maternal infection posed a higher risk for unfavorable outcomes requiring hospitalization and invasive ventilation (Zambrano et al. 2020) and for fetal complications, including preterm birth, growth restriction, and miscarriage (Yee et al. 2020; Barrero-Castillero et al. 2020). These can be

51 mitigated by interventions that strengthen prenatal immunity.

There is a long history of safe and effective immunization of pregnant women against tetanus and diphtheria (Forsyth et al. 2015). In the past decade, the introduction of maternal acellular pertussis vaccine (ACIP 2013) propitiously reduced infant hospitalizations and respiratory infections (Ohfuji et al. 2018; Regan et al. 2016). Seasonal influenza vaccination during pregnancy has been recommended to reduce the risk of infection and severity of disease of mothers and young infants (ACIP 2013; Fiore et al. 2010; Safety 2014). Several studies have demonstrated efficacy of this approach in reducing both maternal and infant hospitalization and illness (Thompson et al. 2019). 
Recognizing the value of this strategy to improve public health, a growing number of vaccine candidates intended to strengthen maternal-infant immunity through vaccination during pregnancy are advancing in the clinical pathway (Saso and Kampmann 2020; Vojtek et al. 2018). influence vaccine-induced immunity. Clinical studies of vaccination in pregnancy have focused primarily on the safety of these new interventions and their immunogenicity, through the analysis of serum antibody levels in mothers and their infants (Monath and Nasidi 1993; Sperling et al. 2012; Schlaudecker et al. 2012; Ohfuji et al. 2018; Munoz et al. 2020). A few of these studies have examined maternal T cell responses (Huygen et al. 2015) and/or functional capacity of vaccine-induced antibodies (Jennewein et al. 2019). However, it remains largely undefined whether the state of pregnancy per se influences vaccine-induced adaptive immunity and if that is the case, what the implications of such changes would be for both maternal and infant health. was to examine the biophysical and functional properties of influenza-specific antibodies in pregnant and non-pregnant women who received seasonal influenza vaccination. We tested the hypothesis that pregnancy-associated structural changes would likely affect both Fab- and non-neutralizing Fc-antibody mediated functions, which are key contributors of protective immunity (Jennewein et al. 2019). To this end, we conducted an in-depth characterization of antibodies to matched seasonal influenza vaccine strains in pregnant and non-pregnant women that included: 1) serum antibody levels determined by hemagglutinin (HA) globular head and stem-specific IgG ELISA, hemagglutination inhibition (HAI) and microneutralization (MN); 2) HA-specific IgG avidity; 3) HA-specific IgG subclasses and Fc receptor binding by ELISA; 4) glycan profile of total and HA-specific IgG Fc and Fab molecules; 5) Fc-mediated

81 innate cell functions: antibody dependent neutrophil phagocytosis (ADNP), antibody dependent

82 monocyte phagocytosis (ADCP), antibody dependent complement deposition (ADCD), and antibody

83 dependent NK cell degranulation; and 6) in vivo analysis of maternal IgG retention. Immunological

84 outcomes in the two groups were compared using multivariate analysis. Distinct profiles of influenza- 
specific IgG Fab and Fc glycan profiles and function were identified between pregnant and nonpregnant vaccine recipients, with subtype-specific antibodies being differentially affected. Two welldefined segregated clusters were identified by aggregate data analysis. These results support similar studies to inform vaccine development and implementation in this important population.

\section{RESULTS}

Pregnancy associated differences in influenza-specific antibody titer

To understand pregnancy-associated modulation of humoral immunity, we conducted a comparative analysis of the magnitude, structural features, and functionality of influenza antibodies in pregnant and non-pregnant women after seasonal influenza vaccination (Supplemental Table 1). stem-specific serum IgG titers (Figure 1A, B) and canonical function of influenza antibodies: hemagglutinin inhibition (HAI) and microneutralization (MN) (Figure 1C, D). Antibody reactivity was tested against two of the subtypes included in the vaccine 2017-18 northern hemisphere flu vaccine ((WHO) 2017): H1 MI (drifted strain of the 2009 H1N1 pandemic lineage which was included since the 2016-17 Northern Hemisphere vaccine--A/Michigan/45/2015, H1N1) and H3 HK (A/Hong

Kong/4801/2014, H3N2), as well as against the original $2009 \mathrm{H} 1 \mathrm{~N} 1$ pandemic strain $\mathrm{H} 1 \mathrm{CA}$

(A/California/07/2009, H1N1) that disproportionally and severely affected pregnant women during the

2009 pandemic (Jamieson et al. 2009; Louie et al. 2010).

Pregnant women had significantly lower IgG titers against H1 MI HA, as compared to the nonpregnant, while titers against H1 CA were unaffected (Figure 1A). A trend of lower H3-lgG was also observed (Figure 1A). Serum IgG directed to the conserved group 1 and group 2 stem regions were both substantially reduced in the pregnant women (Figure 1B). Although both groups had similar HAI and $\mathrm{MN}$ titers for the contemporary vaccine strains $\mathrm{H} 1 \mathrm{MI}$ and $\mathrm{H} 3 \mathrm{HK}$ (Figure 1C, D), pregnant women did exhibit significantly reduced HAl titers against the original pandemic strain H1 CA (Figure 1C). 
Intriguingly, the avidity of IgG against $\mathrm{H} 1 \mathrm{CA} \mathrm{CA}$ was higher in the pregnant women as compared to

111 the non-pregnant (Figure 1E). Likewise, a trend of higher IgG avidity against H1 MI HA was observed.

112 Together, these results hint at pregnancy-associated differences in magnitude, canonical functions, and 113 strength of binding of influenza antibodies, which appear to be antigen-specific.

\section{Pregnancy influences IgG subclass and Fc receptor binding}

116 To better understand the profile of influenza-specific antibodies elicited during pregnancy, we 117 conducted a detailed class ( $\lg$, $\lg A$, and $\lg M)$ and subclass (IgG 1-4 and $\lg \mathrm{A}(1-2)$ analysis of serum 118 antibodies specific for full-length Has $\mathrm{H} 1 \mathrm{MI}$ and $\mathrm{H} 3 \mathrm{HK}$ and their binding capacity to different Fc 119 receptors using a multiplex luminex assay (Figure 2). Consistent with the ELISA data described above 120 (Figure 1A), pregnant women had significantly reduced levels of H1 MI HA-specific total lgG, IgG1, $121 \lg 2$, and IgG3 as compared to non-pregnant women (Figure 2A, B). A similar trend of lower antibody 122 levels (as seen previously by ELISA, in Figure 2) remained for H3 HA-specific IgG and IgG1, and no 123 differences were seen in either $\mathrm{H} 1 \mathrm{MI}$ or $\mathrm{H} 3 \mathrm{HA}$-specific $\lg \mathrm{A} 1$, $\lg \mathrm{A} 2$, or $\lg \mathrm{M}$ levels between the two 124 groups (Figure 2A, B). Pregnant women had a significantly lower ratio of lgG1 and IgG3 to IgG4 for 125 both antigens (Figure 2C, D).

126 Clear differences were also seen in terms of HA antibody binding to Fc-receptors between the 127 two groups (Figure 2E-H). Unlike HA-specific antibody binding patterns revealed by ELISA or luminex 128 assays, both $\mathrm{H} 1 \mathrm{MI} \mathrm{HA}-$ and $\mathrm{H} 3 \mathrm{HK}$ HA-specific antibodies from pregnant women exhibited increased 129 binding to Fc $\gamma \mathrm{R} 1$ as compared to the non-pregnant (Figure 2E). No differences in antibody binding 130 capacity to other FcyR molecules were detected between two groups (Figure 2F-H), except for reduced $131 \mathrm{H} 1 \mathrm{HA}$-specific IgG binding to $\mathrm{Fc} \gamma \mathrm{R} 3$ allotypic variant $\mathrm{V}$ in the pregnant group (Figure 2G). 
examined the Fc- and Fab-glycan composition of total and $\mathrm{H} 1 \mathrm{Ml}$ - and $\mathrm{H} 3 \mathrm{HK}$ - specific serum IgG in both pregnant and non-pregnant women. For each of the samples, a total of 24 distinct glycan peaks were captured by capillary electrophoresis, and overall changes in the four differentially added sugars (galactose, sialic acid, bisecting n-acetyl glucosamine-GlcNAc-and fucose) were calculated for bulk

(Figure 3A, B) and HA-specific (Figure 3C-F, G-V) IgG for each group.

Compared to bulk IgG of non-pregnant women, the bulk Fc glycosylation profile of pregnant women revealed shifts towards increased fucosylation (F) and sialylation (S1 and S2) consistent with an antiinflammatory phenotype (Figure 3A). On the other hand, pregnant women's bulk Fab had fully fucosylated (F), agalactosylated (G0 and G1) and bisected (B) glycans (Figure 3B, O-V). concordant and included digalactosylated (G2) structures with increased sialylation and reduced bisection (Figure 3C-F, G-V) in the pregnant group, whereas agalactosylated, monosialylated and bisected structures were predominant in the non-pregnant group. Taken together, these results point to properties (Fc).

To explore possible associations between Fab and Fc glycosylation, we performed correlation associations between specific glycan types were detected (Figure $\mathbf{3 W}, \mathbf{X}$ ), which suggest coordinated glycosylation of both Fab and Fc molecules. This was particularly evident in the non-pregnant group.

\section{Antigen-specific non-neutralizing antibody functions are selectively affected by pregnancy}

Given the observed differences in magnitude and glycan composition of HA-specific antibodies during pregnancy, we next investigated their Fc mediated effector functions. Responses were measured independently for the two vaccine strains ( $\mathrm{H} 1 \mathrm{Ml}$ and $\mathrm{H} 3 \mathrm{HK}$ ). Monocyte phagocytosis (ADCP) and complement deposition (ADCD) activity of $\mathrm{H} 1 \mathrm{HA}$-specific antibodies were significantly 
161 lower in pregnant women as compared to the non-pregnant women (Figure 4B, C). Intriguingly, no 162 differences were seen in neutrophil phagocytosis (ADNP) and NK degranulation (Figure 4A, D, E), 163 Both H1 HA- and H3 HA-specific antibodies in the pregnant group exhibited reduced cytotoxicity 164 (ADCC) although it did not reach statistical significance (Figure 4F). Overall, a consistent trend towards 165 reduced antibody functionality across FcR-dependent functions was observed in the pregnant women.

166 The predominant changes on $\mathrm{H} 1 \mathrm{HA}-$ specific antibody function further supports pregnancy-associated 167 impact on antigen-specific humoral immunity.

\section{Multivariate signatures of antibodies during pregnancy}

To dissect the differences between antibodies in pregnant and non-pregnant women, a least absolute shrinkage and selection operator and LASSO Partial Least Squares Discriminant Analysis

172 (LASSO-PLSDA) was conducted. This modeling technique allowed the determination of features that 173 contributed most strongly to the group differences, and these predictors were then visualized on the 174 PLSDA plot. This analysis included all the influenza-specific variables assessed (serum IgG, HAI and 175 MN titers, glycosylation, Fc-effector functions, antibody subtypes Fc receptor binding), 79 in total. 176 Readouts from pregnant and non-pregnant groups were separated and orthogonalized along LV1 177 (Figure 5A). The down-selected features were ranked by their importance in separating the pregnant 178 and non-pregnant antibody profile (Figure 5B). The top predictor was H1 HA-specific antibody binding 179 to FcyR1, which was greatly enriched in pregnant women. Another predictor was the lower $\mathrm{H} 1 \mathrm{HA}-$ 180 specific IgG titer in the pregnant group. The remaining predictors were mainly Fc and Fab glycan 181 residues. The groups segregated in antigen-specific antibody galactosylation profiles; anti-inflammatory 182 H3 HA Fc G1B and H1 HA Fc G2S2F glycans were preeminent in pregnant women whereas 183 inflammatory H1 HA Fc-G1F/G1FB and H1 HA Fab-G0 dominated in the non-pregnant group. Thus, Fc 184 glycosylation and Fc receptor binding were the primary driving forces in determining the model. Most of 185 the parameters down-selected with VIP scores were H1MI HA-specific even though $\mathrm{H} 1 \mathrm{MI}, \mathrm{H} 1 \mathrm{CA}$, and 
H3 HK HA-specific readouts were included in the model, reinforcing the notion of antigen dependency

187 of pregnancy-induced antibody changes.

\section{In vivo retention of antibodies produced during pregnancy}

The distinct glycosylation of influenza antibodies in pregnant women begs the question of the purpose of such post-translational modifications, assuming it would ultimately confer some evolutionary advantage to support offspring's health. Our group had shown selective transfer of maternal antibodies with digalactosylated Fc-glycan (Jennewein et al. 2019). These antibodies preferentially bind the FcRn receptor, which not only mediates translocation of IgG across the placenta but also recycling. Thus, we hypothesized that the concomitant increased galactosylation and sialylation of influenza-specific antibodies in pregnant women could allow longer antibody persistence. To address this question, we conducted an adoptive transfer experiment. Pooled serum from both pregnant and non-pregnant women were administered to mice, and antibody levels measured in circulation (Figure 6). Despite starting at the same levels in the recipient mice, IgG from the pregnant group exhibited significantly higher retention in mouse circulation as compared to IgG from the non-pregnant group (Figure 6). This 201 is consistent with the hypothesized prolonged persistence and reduced catabolism.

\section{Discussion}

Seasonal influenza vaccination during pregnancy is recommended by the Advisory Committee on Immunization Practices (Grohskopf et al. 2020) and the American College of Obstetricians and Gynecologists (“ACOG Committee Opinion No. 732: Influenza Vaccination During Pregnancy” 2018).

211 streptococcus, etc.) that are important causes of neonatal and infant death. This approach, however, 
212 faces unique challenges. Fine-tuned regulatory mechanisms are deployed during pregnancy to balance 213 the tolerogenic environment necessary to avoid unhealthy reactions to paternal antigens with the need 214 to engage robust adaptive immunity against microbial threats (Mor and Cardenas 2010; Schumacher, 215 Costa, and Zenclussen 2014). Profound changes in humoral immunity occur during gestation, which 216 include decreased number of peripheral B cells (Watanabe et al. 1997; Faucette et al. 2015), and 217 increased IL-10-secreting regulatory B cells (Rolle et al. 2013). These changes are also expected to 218 impact vaccine-induced immunity.

219 In this study we report, for the first time, distinct binding and functional features of antibodies 220 specific for seasonal influenza vaccine strains produced during pregnancy. Among the most notable is 221 the overt reduction in total $\lg G$ and $\lg G$ subclasses specific for the $\mathrm{H} 1 \mathrm{Ml}$ vaccine strain. HAl titers against $222 \mathrm{H} 1 \mathrm{CA}$ - the original $2009 \mathrm{H} 1 \mathrm{~N} 1$ pandemic strain, were also reduced and, alarmingly, below 223 seroprotective levels. Changes in antibody levels affected primarily those against H1 HA but not those 224 against $\mathrm{H} 3 \mathrm{HA}$. This could be due to immune imprinting, since $\mathrm{H} 1 \mathrm{CA}$-like viruses have continued to 225 circulate globally since the $2009 \mathrm{H} 1 \mathrm{~N} 1$ pandemic $(\mathrm{H} 1 \mathrm{Ml}$ is an antigenically drifted H1 CA-like virus) while 226 the subclade of predominant $\mathrm{H} 3$ virus has changed almost every other year (WHO ; Allen and Ross 227 2018). The history of prior influenza exposure of these women and pre-existing immunity are not known 228 and could have influenced the responses detected (Kosikova et al. 2018). Others have reported reduced 229 HA-specific IgG and IgG subclass responses, as well as HAl titers to seasonal influenza vaccines during 230 pregnancy (Schlaudecker et al. 2018; Schlaudecker et al. 2012; Bischoff et al. 2013). These studies had 231 access to pre-vaccination samples and reported no differences in basal antibody titers between pregnant 232 and non-pregnant women, and yet were able to detect similar reductions in post vaccine immunity, 233 suggesting that the antibody modulations we have observed are intrinsic of pregnancy.

234 Pregnant women had significantly lower $\operatorname{lgG} 1 / \operatorname{lgG}$ and $\operatorname{lgG} 3 / \lg \mathrm{G} 4$ ratios in response to both H1 235 and $\mathrm{H} 3$ vaccine strains, which is consistent with a Th2-type shift of humoral immunity during gestation. $236 \operatorname{lgG} 1$ and $\operatorname{lgG} 3$ are the main subclasses produced in response to viral antigens (Hjelholt et al. 2013), 237 hence their reduced proportions raise concerns about insufficient protection against viral infections. 
In addition to lower $\lg \mathrm{G}$ response to the $\mathrm{H} 1$ vaccine strain, pregnant women had reduced $\lg \mathrm{G}$ titers against highly conserved group 1 and group 2 HA stem regions. This observation is new and suggests that production of stem-specific antibodies during pregnancy might be impaired and that these antibodies could be limited in their capacity to recognize emerging virus variants. Further studies to understand HA stem-specific immunity in this group are warranted as stem antibodies are hypothesized to confer broad cross-protection (Wu and Wilson 2017) and are candidate antigens for sought after 244 universal flu vaccines (Sautto, Kirchenbaum, and Ross 2018).

Intriguingly, we found that $\mathrm{H} 1 \mathrm{HA}-$ specific IgG in pregnant women had higher avidity compared to

246 those from non-pregnant women. In addition, HA-specific antibodies produced during pregnancy had

247 increased capacity to bind FcyR1. FcyR1 is an activating receptor expressed in DC and 248 monocyte/macrophages, with high affinity for human monomeric lgG, particularly lgG1 (Stewart et al. 249 2014; Bruhns et al. 2009). On the other hand, H1 HA-specific IgG from pregnant women had reduced 250 binding capacity to FcyRIIla-V, a high affinity allotype expressed in monocytes/macrophages. The 251 enhanced capacity of antibodies to bind cognate antigens and to selectively engage in innate cell function 252 via Fc-interaction may represent compensatory mechanisms to maximize response yet avoid harmful 253 effects.

Another novel aspect of our study was the analysis of extra-neutralizing FcR-mediated antibody 255 functions in pregnant women. $\mathrm{H} 1$-specific ADCP, ADCD, and ADCC were reduced in this group, and this 256 observation is consistent with reduced levels of $\mathrm{H} 1 \mathrm{IgG}$, and in particular IgG1, which triggers these 257 processes efficiently by engaging FcyRI (Gunn and Alter 2016). The reduced capacity of H1 HA 258 antibodies to bind to FcyRIII-V, which is expressed in monocytes and macrophages might have also 259 contributed to this outcome. IgG1 has been implicated in activation of complement in the context of 260 influenza infection, hence reduced IgG1 in this group is consistent with lower C3 deposition.

261 Unlike FcR-mediated monocyte/macrophage uptake, neutrophil phagocytic activity and NK 262 degranulation both appeared largely unaffected during pregnancy. Neutrophil phagocytosis can be 
263 activated by different and additional signals such as IgG2 interaction with FcyRlla and IgA with FcaRI

264 (Gunn and Alter 2016). NK degranulation was maintained in the pregnant group despite their antibodies

265 exhibiting lower binding affinity for FcyRIIla (the cellular receptor that mediates NK degranulation). These

266 observations further support the idea of compensatory mechanisms of the humoral immune system to

267 offset pregnancy-intrinsic changes and maintain a healthy state.

268 Vastly different glycosylation of total and influenza-specific antibodies was observed in pregnant

269 women compared to non-pregnant controls. The total Fc glycan profile in pregnant women, which

270 included fucosylation and sialylation, was oriented towards reduced inflammation. In contrast, total Fab

271 glycans, which involved increased fucosylation, agalactosylation, and bisection, were associated with

272 increased inflammation. Non-inflammatory Fc galactosylation and sialylation as well as Fab N-

273 acetylglucosamine bisection of bulk antibodies during pregnancy have been reported elsewhere (Bondt

274 et al. 2014; Jansen et al. 2016; Bondt et al. 2016; Ruhaak et al. 2014; van de Geijn et al. 2009; Selman

275 et al. 2012). Surprisingly, the Fc and Fab glycan profile of HA-specific antibodies were largely

276 concordant within each group and included digalactosylated (G2) structures with increased sialylation

277 and reduced bisection in the pregnant group, whereas agalactosylated, monosialylated, and bisected

278 structures were predominant in the non-pregnant group. The association patterns remained broadly

279 unchanged in the two groups, which indicates a coordinated global regulation of Fc and Fab

280 glycosylation. However, other factors governing production of Fab and Fc glycans may reduce the

281 overall level of coordination to reflect the putative greater level of Fab glycosylation during pregnancy

282 (van de Bovenkamp et al. 2016).

283 Two distinct populations emerged in the PLSDA analysis, with Fc and Fab glycosylation being

284 primary discriminatory elements; the clear separation of the two groups confirms the robustness of the

285 data. The similarity in Fc and Fab glycosylation for the influenza-specific antibodies suggests some

286 level of coordination of post-translational modifications in the Golgi that differs according to the B cell

287 population. This is the first demonstration of Fab and Fc glycosylation of vaccine-specific antibodies 
consistent with non-inflammatory activity in pregnant women and different from the more inflammatory pattern in the non-pregnant group. Despite the disparity in antibody glycosylation, all the relevant Fcmediated influenza-specific cellular functions were detected in the pregnant group.

We have previously shown that digalactosylated antibodies involved in NK-degranulation were enriched in cord blood; these digalactosylated antibodies preferentially bind to the neonatal Fc receptor 293 (FcRn), which transits antibodies across the placenta (Jennewein et al. 2019; Martinez et al. 2019). 294 Digalactosylated antibodies are reportedly more effective at facilitating NK-degranulation, a function the 295 neonatal immune system is better equipped to deploy as opposed to phagocytosis or other nonneutralizing functions (Jennewein et al. 2019). For the mother, digalactosylation of antibodies during gestation may reflect another compensatory mechanism that maintains ADNP unaltered when other cellular functions may be reduced. Likewise, sialylation has been exploited in immunoglobulin therapy to endow anti-inflammatory benefits (Anthony et al. 2008; Pagan, Kitaoka, and Anthony 2018; Bruckner et al. 2017). Here, enhanced Fc-sialylation during pregnancy may contribute to dampened antibodymediated inflammation consistent with the tolerizing state of pregnancy (Pagan, Kitaoka, and Anthony 2018).

Lastly, serum antibodies from pregnant women were retained longer in mouse circulation as demonstrated in in vivo passive transfer experiments, suggesting that beyond impacting the inflammatory state, the biophysical changes described above, in particular concomitant increased galactosylation and sialylation (Bas et al. 2019) may be intended to ensure sufficient circulating antibody during gestation during extended periods for protection of both the mothers and newborns. Together, the shifts observed in influenza-specific antibodies during pregnancy are consistent with reduced inflammation, more efficient Fc receptor engagement to maintain anti-viral function (as the magnitude of antibody available might be 310 reduced), enhancement of placental transfer, reduction in antibody catabolism, and enrichment of 311 antibodies that are more efficient for the newborn. The origin, regulation, and evolution of these 312 pregnancy-associated antibody changes remains to be investigated. 
Rather than broad immune suppression during pregnancy (Memoli et al. 2013), our data suggest

314 that humoral immunity is selectively modulated by changes in antibody levels, avidity, FcR binding 315 capacity, glycosylation patterns, and innate cellular functions. Our results also argue against universally 316 compromised humoral immunity and in favor of discrete, antigen-dependent modifications.

317 While our study is limited by a small sample size and the retrospective analysis of immune 318 responses to influenza seasonal vaccine strains, it still provides a valuable snapshot of vaccine-specific 319 immunity in the pregnant population. Maternal infections place women and their infants at high risk. 320 Understanding pregnancy-associated changes in antibody production, post-translational modifications 321 and function, as well as how these processes affect vaccine responses will benefit vaccine design and 322 will inform the development of much needed preventive and therapeutic strategies, particularly those for 323 use in emergency situations, to improve maternal and infant health.

\section{Acknowledgments}

326 H. X. was supported by the fund of FDA Office of Women's Health.

Author contributions:

329 Conceptualization, M.F.J., H.X., G.A., and M.F.P.; Clinical samples: M.F.P, W.H.C and J.D. C. 330 Investigation; M.F.J., M.K., F.J.N., P.R., and C.M.B.; Resources, H.X., G.A., and M.F.P.; Writing-Original 331 draft, M.F.J, H.X., G.A., and M.F.P.; Writing-review and editing, J.D.C, W.H.C.; Supervision, H.X., G.A., 332 and M.F.P.

Declaration of interests:

335 The authors have no competing interests to declare 
Figure Legends:

Figure 1: Pregnancy associated differences in influenza-specific antibody titer. HA-specific IgG (A), group 1- and group 3-stem specific IgG (B), hemagglutination inhibition (HAI) (C), microneutralization (MN) (D) and HA-specific IgG avidity (E) in pregnant and non-pregnant women. Data represent log transformed individual datapoints and geometric mean titers (bars). ELISA, HAI and MN titers were compared using one-tailed Mann-Whitney test. Avidity was evaluated using a two-tailed Mann-Whitney test ${ }^{*} p<0.05,{ }^{* *} p<0.01$.

\section{Figure 2: Pregnancy influences HA-specific IgG subclass and Fc receptor binding.} $\lg G$ and $\lg G$ subclass levels and the Fc receptor binding were assessed by Luminex. A-B. Dot plots depict the differences between isotype and subclass levels for non-pregnant (NP, grey circles) and pregnant (P, blue circles) women. A. Isotypes. B. IgG subclasses. C-D. IgG1/lgG4 (C) lgG3/lgG4 ratios (D). E-H. Class 1 Fc receptor. D. Class 2 Fc receptors. E. Class 3 Fc receptors. F. Neonatal Fc receptor. Statistics evaluated as Mann-Whitney test and stars represent differences between $\mathrm{H} 1$ and $\mathrm{H} 3$ response.

Figure 3: Pregnancy alters antibody Fc and Fab glycosylation.

355 Antibody glycosylation for HA-specific and bulk antibodies was analyzed by capillary electrophoresis.

356 A-R. Radar plots depicting different glycosylation profiles of bulk IgG Fc (A) and Fab (B), H1-specific $357 \operatorname{IgG} \mathrm{Fc}(\mathrm{C})$ and $\mathrm{Fab}(\mathrm{E})$ and H3-specific IgG Fc (D) and Fab (E) in non-pregnant and pregnant groups.

$358 \mathrm{G}=$ galactose (0-2), F=fucose, B=Bisecting GlcNAc, S=sialic acid (1-2). G-V. These dot-line and stacked-bar plots depict the differences in HA1-specific antibody Fc (G-J) and Fab (O-R) and HA3specific antibody Fc (K-N) and Fab (S-V) glycosylation between non-pregnant (NP) and pregnant (P) women. (W, X) Heatmaps showing the spearman correlation between HA-specific Fab and Fc glycosylation for pregnant (W) and non-pregnant (X) women. Significance for radar plots and glycosylation determined by Mann-Whitney test. ${ }^{*} p<0.05,{ }^{* *} p<0.01,{ }^{* * *} p<0.001,{ }^{* * * *} p<0.0001$. 
Figure 4: Antigen-specific non-neutralizing antibody functions are affected by pregnancy.

Influenza H1- and H3-specific antibody dependent neutrophil phagocytosis (ADNP) (A), monocyte

(B), complement deposition (ADCD) (C), NK degranulation measured as

CD107a, MIP-1 $\beta$ and IFNy (D, E) and cellular cytotoxicity (ADCC) (F). Groups were compared using two-tailed Mann-Whitney Test. ${ }^{*} p<0.05,{ }^{* *} p<0.01$.

Figure 5: Multivariate signatures of antibodies produced during pregnancy.

372 Computational analysis was used to distinguish features enriched in pregnancy. A. LASSO Partial

373 Least Squares Discriminant Analysis (LASSO-PLSDA) of influenza-specific antibody features between

374 pregnant and non-pregnant women orthogonalized along latent variable 1 (LV1). LV1 explains $42.5 \%$ of

375 the variance along the $X$ axis while LV2 explained $17.1 \%$ of the variance. B. The Variable Importance in

376 Projection (VIP) scores for the PLSDA indicate the prime factors driving the differences between

377 pregnant and non-pregnant women. Factors pointing towards the left are enriched in non-pregnant

378 samples, while those pointing right are enriched in pregnant samples. Bar color and length corresponds

379 to relative importance. Antibody features are colored by category.

\section{Figure 6. In vivo antibody retention.}

382 The dot-line plot depicts the retention of IgG in mice injected with pooled plasma from pregnant (blue, 4 383 mice) or non-pregnant (grey, 5 mice) mice. Retention was measured over a 6-day period. Dots are at 384 mean with error bars at the standard deviation. Statistics evaluated using a two-way ANOVA. * $p<0.05$, $385{ }^{* *} \mathrm{p}<0.01$.

\section{Supplemental Figure 1: Relationship of antibody profiles and vaccination.}

PCA analysis was used to distinguish effects of vaccination. No differences were observed across the antibody profiles for vaccination and un-vaccinated participants. Principle component 1 (PC1) contains $12 \%$ of the variance and PC2 contains $10.5 \%$ of the variance. A. Dots colored to distinguish pregnant (blue dots) and non-pregnant (grey dots) women. B. Dots colored to distinguish women who received seasonal influenza vaccine (pink dots) and women without clinical record of vaccination (orange dots). 
398 Supplemental Table 1: Demographics of study participants

399

\begin{tabular}{|c|c|c|}
\hline & Non-pregnant & Pregnant \\
\hline Age & $\begin{array}{l}31.35 \\
\text { (Ranqe: 23-44) }\end{array}$ & $\begin{array}{l}27.64 \\
\text { (Ranae: 18-35) }\end{array}$ \\
\hline Hormonal birth control & $\begin{array}{l}10: 10 \\
\text { (yes: no) }\end{array}$ & N/A \\
\hline Received flu vaccine & $\begin{array}{l}\text { 15:5 } \\
\text { (yes:no record) }\end{array}$ & $\begin{array}{l}\text { 11:3 } \\
\text { (yes:no record) }\end{array}$ \\
\hline $\begin{array}{r}\text { Received TDAP } \\
\text { vaccine }\end{array}$ & $\begin{array}{l}0: 20 \\
\text { (yes:no) }\end{array}$ & $\begin{array}{l}\text { 14:0 } \\
\text { (yes:no) }\end{array}$ \\
\hline $\begin{array}{r}\text { Months between } \\
\text { vaccine and sample } \\
\text { collection }\end{array}$ & $\begin{array}{l}4.2 \\
\text { (Range: 0-6) }\end{array}$ & $\begin{array}{l}4.3 \\
\text { (Range:2-6) }\end{array}$ \\
\hline
\end{tabular}

400

401 


\section{METHODS}

404 Human samples. Serum samples were obtained from pregnant (end of $3^{\text {rd }}$ trimester) and non-pregnant 405 women who received egg-based inactivated influenza vaccine during the 2017-2018 flu season;

406 samples were obtained within 4 months of vaccination (Supplemental table 1, Supplemental figure

407 1). The vaccine components for the northern hemisphere $2017-2018$ season influenza vaccine included 408 H1N1 A/Michigan/45/2015, H3N2 A/Hong Kong/4801/2014, B/Phuket/3073/2013, and

409 B/Brisbane/60/2008-like viruses((WHO) 2017). All subjects were HIV, HBV, and HCV negative and 410 provided written informed consent before enrollment. This study was approved by the University of 411 Maryland, Baltimore Institutional Review Board (HP-00065842 and HP-00040025) and by Partners 412 Human Research Committee (Approval Number 2018P001060).

Cell lines. Madin-Darby canine kidney-SIAT1 cells (SIAT1-MDCK, Sigma Aldrich, St. Louis, MO, USA), were grown in MEM plus $50 \mu \mathrm{g} / \mathrm{ml}$ of $\mathrm{G} 418,5 \%$ fetal bovine serum, $1 X$ GlutaMAX $^{\mathrm{TM}}$ and penicillin/streptomycin. THP-1 cells (ATCC, Manassas, VA, USA), were grown in R10 (RPMI plus 10\% FBS L-glutamine and penicillin/streptomycin) supplemented with $0.01 \% \beta$-mercaptoethanol.

Viruses. H1N1 A/California/07/2009 (H1 CA), H1N1 A/Michigan/45/2015 (H1 MI), and H3N2 A/Hong Kong/4801/2014 (H3 HK) were grown in 9-10 day old embryonated eggs. Infectious viral titer, i.e., 50\%

421 tissue culture infectious dose (TCID50), was determined using a nucleoprotein-based enzyme-linked 422 immunosorbent assay (ELISA) (Kosikova et al. 2018).

424 Recombinant proteins. For titer and avidity determination, genes coding H3 HK HA, chimeric HA 425 bearing $\mathrm{H} 1 \mathrm{~A} /$ Puerto Rico/8/1934 stalk (group 1) with mismatched H16 HA head and chimeric HA bearing H3 HK stalk (group 2) with mismatched H4 HA head were synthesized as previously described 
427 (Stevens et al. 2004; Pica et al. 2012; Radvak et al. 2021) and were subcloned into the pFastBac gp67 428 vector (GenScript, Nanjing, China). Recombinant H3 HK HA, chimeric group 1 or group 2 stalk proteins 429 expressed in Sf9 cells were column purified (GenScript). For functional assays and luminex Fc receptor 430 binding and titer, recombinant $\mathrm{H} 1 \mathrm{MI}(\mathrm{HA} \triangle \mathrm{TM} \mathrm{H} 1 \mathrm{~N} 1 \mathrm{~A} /$ Michigan/45/2015), H1 CA (HA $\triangle \mathrm{TM}$

431 A/California/07/2009) and H3 HK proteins (H3ATM H3N2 A/Hong Kong/4801/2014) expressed in 293 432 cells were purchased from Immune Technology Corp. (New York, NY, USA).

434 Anti-HA ELISA and avidity. HA-specific ELISA and avidity assays were performed as described 435 (Kosikova et al. 2018). Briefly, serum samples were serially diluted and added to MaxiSorp microtiter 436 plates (ThermoFisher) coated with $0.2 \mu \mathrm{g} / \mathrm{mL}$ of recombinant protein. Plates were incubated at room 437 temperature for 60 min. For avidity determination, antigen-antibody complexes were dissociated by 438 incubation with $100 \mu \mathrm{l}$ of $4 \mathrm{M}$ urea for 15 minutes and plates blocked again for an additional hour 439 (Kosikova et al. 2018). Bound antibodies were detected by addition of horseradish peroxidase-labeled 440 goat-anti-human IgG (Invitrogen, Carlsbad, CA, USA) followed by 1-Step Ultra TMB (ThermoFisher) 441 substrate. Absorbance at $450 \mathrm{~nm}$ was measured in a Victor $\mathrm{V}$ multilabel reader (PerkinElmer, Waltham, 442 MA, USA). Antibody titers were determined by interpolation in a Sigmoidal four parameter logistic 443 regression curve. Avidity index was calculated based on the area of the entire antibody titration curve 444 as described previously (Kosikova et al. 2018).

446 Hemagglutination inhibition (HAI). Sera were pre-treated with receptor-destroying enzyme (RDE) 447 (Denka-Seiken, Tokyo, Japan), serially diluted two-fold (starting at 1:10) and incubated with 4 HA units 448 per $25 \mu \mathrm{l}$ of viruses at room temperature for $30 \mathrm{~min}$. Hemagglutination was determined using $0.5 \%$ 449 turkey red blood cells for $\mathrm{H} 1 \mathrm{~N} 1$ viruses or $0.75 \%$ guinea pig red blood cells in the presence of $20 \mathrm{nM}$ 450 oseltamivir for H3N2 viruses as described previously (Xie et al. 2015; Xie et al. 2011). HAl titers were 451 expressed as the reciprocal of the highest serum dilution that resulted in complete HAI. A titer of 5 was 
assigned if no inhibition was observed at the starting dilution.

Microneutralization (MN). H1N1 and H3N2-specific MN titers were determined using a nucleoproteinbased ELISA as previously described (Kosikova et al. 2018). Briefly, RDE-treated sera were incubated with $10050 \%$ tissue culture infectious dose $\left(\mathrm{TCID}_{50}\right)$ of virus for $1 \mathrm{~h}$ at room temperature, $5 \% \mathrm{CO}_{2}$, and then overlaid onto SIAT1-MDCK cells (Sigma) overnight. Infected cells were detected using influenza A nucleoprotein-specific monoclonal antibodies (Millipore, Burlington, MA, USA). MN titers were reported as the reciprocal of the highest serum dilution with $\geq 50 \%$ neutralization.

Phagocytosis. The antibody-dependent monocyte phagocytosis assay (ADCP) used was adapted from Ackerman et al. 2011 and the antibody-dependent neutrophil phagocytosis assay (ADNP) was performed as described (Karsten et al. 2019). Briefly, influenza antigens, recombinant HA $\triangle T M H 1 N 1$ A/Michigan/45/2015 and HA $\triangle T M$ H3N2 A/Hong Kong/4801/2014 were biotinylated with a 20-fold excess of 10 mM EZ-Link NHS-LC-biotin (ThermoFisher) and unbound biotin was removed using a Zeba Spin Desalting Column (ThermoFisher). Antigens were then mixed with yellow-green fluorescent NeutrAvidin microspheres (ThermoFisher) at a ratio of $10 \mu \mathrm{l}$ of beads per $10 \mu \mathrm{g}$ of biotinylated protein and incubated at $37^{\circ} \mathrm{C}, 5 \% \mathrm{CO}_{2}$ for 2 hours. Following incubation, beads were washed twice with PBS$0.01 \%$ BSA and resuspended in $1 \mathrm{~mL}$ of PBS-0.01\% BSA. Serum samples were diluted 1:10, 1:50, and 1:100 in PBS, and $10 \mu \mathrm{l}$ of each dilution was mixed with $10 \mu \mathrm{l}$ of antigen-coupled fluorescent beads in a

47196 -well round bottom plate and incubated at $37^{\circ} \mathrm{C}, 5 \% \mathrm{CO}_{2}$, for $2 \mathrm{~h}$. The beads were then washed with $472200 \mu$ l of PBS. For the monocyte phagocytosis, THP-1 cells $\left(2.5 \times 10^{4}\right.$ cells in $200 \mu$ l per well $)$ were 473 added to wells containing the beads, and plates were incubated for $16 \mathrm{~h}$ at $37^{\circ} \mathrm{C}, 5 \% \mathrm{CO}_{2}$. For the 474 neutrophil phagocytosis, fresh neutrophils were isolated from whole blood of healthy adults and added $475\left(5 \times 10^{5}\right.$ cells in $\left.200 \mu \mathrm{l}\right)$ to bead-containing wells. Plates were incubated for $1 \mathrm{~h}$ at $37^{\circ} \mathrm{C}, 5 \% \mathrm{CO}_{2}$. 476 Following incubation, cells were spun down and stained with anti-CD66b (clone:G10F5, BioLegend, 
477 Dedham, MA, USA) for 15 min. For both assays, cells were fixed with $100 \mu$ l of $4 \%$ paraformaldehyde

478 (PFA) for $10 \mathrm{~min}$, resuspended in $50 \mu \mathrm{l}$ of PBS and analyzed by Flow cytometry using an iQue

479 Screener PLUS (IntelliCyt, Albuquerque, NM, USA) and ForeCyt software (IntelliCyt). Influenza-specific

$480 \lg$ and PBS were included as positive and negative controls. Phagocytosis scores were calculated as

481 the percentage of bead positive cells, multiplied by the geometric mean fluorescent intensity of the

482 beads for bead positive cells, divided by $1 \times 10^{7}$. All reported values are the area under the curve of the

483 mean of two replicates.

Complement deposition. Antibody-dependent complement deposition (ADCD) was performed as

486 previously described (Fischinger et al. 2019). Biotinylated recombinant HA $\triangle T M H 1 N 1$

\section{7}

488

489

A/Michigan/45/2015 and H3ATM H3N2 A/Hong Kong/4801/2014 were coupled to red fluorescent

NeutrAvidin microspheres (ThermoFisher), incubated with sera and added to single wells as described above. Lyophilized guinea pig complement (Cedarlane, Burlington, ON, Canada) was resuspended in ice-cold water and diluted 1:100 in cold veronal buffer supplemented with $0.1 \%$ fish skin gelatin (Boston Bio Products, Boston, MA, USA). $200 \mu$ l of diluted complement was added to each well, and plates were incubated for $20 \mathrm{~min}$ at $37^{\circ} \mathrm{C}, 5 \% \mathrm{CO}_{2}$. Plates were then washed twice with ice-cold $15 \mathrm{mM}$ EDTA-PBS and stained with anti-guinea pig C3-FITC (MP Biomedical, Santa Ana, CA, USA). After a final wash, beads were re-suspended in $50 \mu \mathrm{l}$ of PBS and analyzed on the iQue Screener PLUS. Redfluorescent beads were gated based on size. Complement deposition was determined as the geometric mean fluorescent intensity of red fluorescence of the beads divided by 1,000 . Data presented is area under the curve of the complement score averaged for two replicates.

NK cell Activation. ELISA plates were coated with recombinant HA $\triangle T M H 1 N 1$ A/Michigan/45/2015 and $\mathrm{H} 3 \Delta \mathrm{TM} H 3 \mathrm{~N} 2 \mathrm{~A} / \mathrm{Hong}$ Kong/4801/2014 at $3 \mu \mathrm{g} / \mathrm{mL}$ of PBS for $2 \mathrm{~h}$ at $37^{\circ} \mathrm{C}, 5 \% \mathrm{CO}_{2}$. Plates were then washed three times with PBS and blocked overnight with $200 \mu \mathrm{l}$ of $5 \%$ BSA in PBS. The next day, plates 
were washed, and samples were added in three serial dilutions $(1: 10,1: 50,1: 100)$ in a $50 \mu$ volume and

503 incubated $2 \mathrm{~h}$ at $37^{\circ} \mathrm{C}, 5 \% \mathrm{CO}_{2}$. NK cells were isolated from healthy adult PBMC using a RosetteSep NK

504 Cell Enrichment Kit (Stem Cell Technologies, Vancouver, BC, Canada), according to manufacturer's 505 instructions followed by density gradient centrifugation in Histopaque. A suspension of $1.5 \times 10^{6} \mathrm{cells} / \mathrm{ml}$ 506 in R10 was prepared, supplemented with $1 \mathrm{ng} / \mathrm{ml}$ rhlL15 and incubated overnight at $37^{\circ} \mathrm{C}, 5 \% \mathrm{CO}_{2}$. Prior 507 to the assay, a suspension of $2.5 \times 10^{5} \mathrm{cells} / \mathrm{ml}$ was prepared and $2.5 \mu \mathrm{g} / \mathrm{ml}$ of Brefeldin A (Biolegend) 508 and Golgistop (BD Biosciences, Franklin Lake, NJ, USA) and $2.5 \mu$ l of anti-CD107a (Clone:555802, BD 509 Biosciences) were added. NK cells $\left(5 \times 10^{4}\right.$ in $\left.200 \mu l\right)$ were added to each well containing Ag-Ab, and plates 510 were incubated for $5 \mathrm{~h}$ at $37^{\circ} \mathrm{C}, 5 \% \mathrm{CO}_{2}$. Following the incubation and washing, cells were stained with 511 anti-CD3 (Clone:UCHT, BD Biosciences), anti-CD56 (Clone: B159, BD biosciences), and anti-CD16 512 (Clone: 3G8, BD biosciences), washed, fixed, permeabilized with FIX\&Perm kit (ThermoFisher), and 513 stained intracellularly with anti-IFNy (Clone:B27, BD biosciences) and MIP-1 $\beta$ (Clone:D21-1351, BD 514 Biosciences). Cells were resuspended in PBS and analyzed on the iQue Screener as described above. 515 Influenza-specific IgG and PBS (diluent) were included as positive and negative controls. Negative control wells were used to set gates. Data was reported as the percentage of CD16+CD56+CD3- NK 517 cells positive for CD107a, IFNy, or MIP-1 $\beta$ and is the area under the curve of the mean of three replicates 518 divided by 100,000 .

Cellular cytotoxicity. Antibody-dependent cellular cytotoxicity (ADCC) assay was performed as 521 previously described (Jacobsen et al. 2017) with modifications. Briefly, SIAT1-MDCK cells were seeded 522 onto 96-well white flat-bottom plates (PerkinElmer), infected with $\mathrm{H} 1 \mathrm{Ml}$ or $\mathrm{H} 3 \mathrm{HK}$ viruses and incubated 523 for $6 \mathrm{~h}$ at $37^{\circ} \mathrm{C}$. RDE-treated sera $(25 \mu \mathrm{l} /$ well) and Jurkat effector cells (Promega, Madison, WI, USA) $524(25 \mu \mathrm{l} /$ well $)$ were added to infected cells and incubated overnight at $37^{\circ} \mathrm{C}$. Bio-Glo luciferase assay 525 reagent (Promega) was then added, and luminescence was measured using a Victor $\vee$ multilabel reader (PerkinElmer). 
528 Antibody subclass and FcR binding. $\lg$, $\lg A$, $\lg M$, and Fc receptor binding analyses were

529 performed using a multiplex luminex assay as previously described (Brown et al. 2012). Briefly,

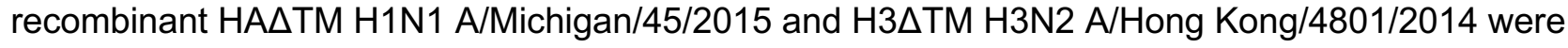

531 coupled to MagPlex microspheres (Luminex corporation, Austin, TX, USA) at a ratio of $25 \mu \mathrm{g}$ of protein to $400 \mu$ l of beads. Microspheres were activated with $100 \mathrm{mM}$ monobasic sodium phosphate $\mathrm{pH} 6.2$ in the presence of $50 \mathrm{mg} / \mathrm{mL}$ EDC and $50 \mathrm{mg} / \mathrm{ml}$ sulfo-NHS, washed with $0.05 \mathrm{M} 2[\mathrm{~N}-$ Morpholino]ethanesulfonic acid (MES) $\mathrm{pH} 5.0$, incubated with antigen for $2 \mathrm{~h}$ at $37^{\circ} \mathrm{C}, 5 \% \mathrm{CO}_{2}$, and blocked with PBS-TBN (0.1\% BSA, 0.02\% tween-20, 0.05\% Azide in PBS, pH 7.4) for 30 min. The beads were washed in PBS- $0.05 \%$ tween-20, resuspended in $250 \mu$ of PBS. Beads were added to 384-well plates and incubated with sera at a final 1:500 dilution in luminex wash buffer (PBS-0.05\% BSA-0.001\% tween-20) for $2 \mathrm{~h}$ at room temperature and with shaking (800 rpm).

To detect antibody subclasses, following incubation, beads were washed in luminex buffer and 540 incubated with PE-labeled anti-lgG, - $\lg G 1,-\lg G 2,-\lg G 3,-\lg G 4,-\lg A 1,-\lg A 2$, and - $\lg M$ (Southern 541 Biotech, Birmingham, AL, USA) for $1 \mathrm{~h}$ at room temperature with shaking. After washing, beads were 542 resuspended in Qsol buffer (IntelliCyte) and plates were read on an iQue Screener PLUS. H1N1 and 543 H3N2 coupled beads were distinguished based on size and granularity, and data were analyzed as median fluorescent intensity of PE for each bead population.

545 To investigate Fc receptor binding recombinant Fc receptors with an AviTag were biotinylated using 546 a Bir500 kit (Avidity, Aurora, CO, USA) according to manufacturer's instructions and purified using a Zeba 547 Spin Desalting Column. Biotin-labeled FcRs were then incubated with streptavidin-PE (Prozyme, 548 Hayward, CA, USA) for $10 \mathrm{~min}$ and excess streptavidin was quenched with $20 \mu \mathrm{M}$ biotin (Avidity) for 549 another 10 min. Following bead and serum incubation, PE-Fc receptors were then added and incubated 550 for a further $1 \mathrm{~h}$ at room temperature with shaking. Plates were then washed and analyzed on an iQue 551 Screener as described above. Data reported for each sample represents the mean of two replicates. 
553 Glycosylation analysis. Antigen-specific glycosylation analysis was performed as described in

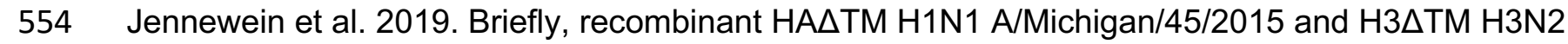
$555 \mathrm{~A} /$ Hong Kong/4801/2014 were biotinylated and coupled to streptavidin magnetic beads (New England 556 Biolabs) at a ratio of $2.5 \mu \mathrm{g}$ of protein to $25 \mu \mathrm{l}$ of beads. Sera $(200 \mu \mathrm{l})$ were first incubated with non557 coated NeutrAvidin beads to remove non-specific binding and then added to the antigen-coated beads 558 and incubated for $1 \mathrm{~h}$ at $37^{\circ} \mathrm{C}$. Antibodies were eluted by incubation in $50 \mu \mathrm{l}$ of $\mathrm{pH} 2.9$ citrate buffer for $55930 \mathrm{~min}$ at $37 \mathrm{C}$. Samples were then spun down and the eluted antibodies, contained in the supernatant, 560 were neutralized with $30 \mu \mathrm{l}$ H 8.9 potassium phosphate buffer. Antibodies were then coupled to 561 protein G beads (New England Biolabs). After beads were washed, IDEZ was used to cleave the Fab 562 (in the supernatant) from the Fc (remained on the magnetic beads) (New England Biolabs) for $1 \mathrm{~h}$ at $56337^{\circ} \mathrm{C}$ and collected. The two fragments were deglycosylated and fluorescently labeled using a 564 GlycanAssure APTS kit (ThermoFisher scientific) according to manufacturer's instructions. For the Fc 565 fragment, which remained bound to the protein $\mathrm{G}$ beads, an additional magnetic separation after 566 PNGase glycan cleavage separated the glycans from remaining magnetic beads and the protocol then 567 proceeded per manufacturer's instructions. Glycans were analyzed on a 3500xL genetic analyzer. 568 Glycan fucosyl and afucosyl libraries (Prozyme) were used to assign 24 discrete glycan peaks using 569 GlycanAssure software (ThermoFisher). Data are reported as percentages of total glycans for each of 570 the glycan peaks.

571

572 In vivo Ab retention. Pooled sera from pregnant and non-pregnant women were transferred 573 intraperitoneally into naïve female Balb/c mice (Charles River, Wilmington, MA) at $0.2 \mathrm{ml} / \mathrm{mouse}$. The 574 circulating concentration of human IgG after passive transfer was measured by a Human lgG Easy 575 Quantification ELISA Kit (Cell Sciences, Newburyport, MA). A human IgG calibrated standard was 576 included for calculation of IgG content. 
577 Statistical Analysis. Mann-Whitney test (univariate analysis) was used to examine differences in 578 readouts between pregnant and non-pregnant groups. $P$ values are two-sided, ${ }^{*} p<0.05,{ }^{* *} p<0.01$, $579{ }^{* * *} p<0.001$, and ${ }^{* * *} p<0.0001$. Data on radar plots were z-score normalized individually along each 580 radar to an average of 0 with a standard deviation of 1 . Multivariate Principal Component 581 Analysis (PCA) and Least absolute shrinkage and selection operator LASSO Partial Least Squares 582 Discriminant Analysis (LASSO-PLSDA) were used to distinguish pregnant vs. non-pregnant immune 583 profiles. LASSO is a regression analysis that selects variables that contribute to the differences 584 between samples arranged along an ordinal dependent variable. It selects features that are required to 585 create a maximum separation which are then visualized on the PLSDA plot. Missing values were 586 imputed using the k-nearest neighbor. Variables were centered and scaled to a standard deviation of 1. 587 PCA was used to visualize if pregnant and non-pregnant samples separate into discrete groups within 588 the two-dimensional principal component (PC) space. The LASSO-PLSDA model had a calibration 589 success of $100 \%$ with a leave-1-out CV success of $97.0588 \%$. Univariate analysis was performed with 590 Prism 7 (GraphPad, San Diego, CA). MATLAB was used to perform all multivariate analysis. 


\section{References}

World Health Organization. 2017. "Recommended composition of influenza virus vaccines for use in the 2017-2018 northern hemisphere influenza season." In. Gneva.

ACIP. 2013. "Updated Recommendations for Use of Tetanus Toxoid, Reduced Diphtheria Toxoid, and Acellular Pertussis Vaccine (Tdap) in Pregnant Women." In, 131-35. Morbidity and Mortality Weekly Report: CDC.

Ackerman, M. E., B. Moldt, R. T. Wyatt, A. S. Dugast, E. McAndrew, S. Tsoukas, S. Jost, C. T. Berger, G. Sciaranghella, Q. Liu, D. J. Irvine, D. R. Burton, and G. Alter. 2011. 'A robust, high-throughput assay to determine the phagocytic activity of clinical antibody samples', J Immunol Methods, 366: 8-19.

'ACOG Committee Opinion No. 732: Influenza Vaccination During Pregnancy'. 2018. Obstet Gynecol, 131: e109-e14.

Allen, J. D., and T. M. Ross. 2018. 'H3N2 influenza viruses in humans: Viral mechanisms, evolution, and evaluation', Hum Vaccin Immunother, 14: 1840-47.

Anthony, R. M., F. Nimmerjahn, D. J. Ashline, V. N. Reinhold, J. C. Paulson, and J. V. Ravetch. 2008. 'Recapitulation of IVIG anti-inflammatory activity with a recombinant IgG Fc', Science, 320: 373-6.

Barrero-Castillero, A., K. S. Beam, L. B. Bernardini, E. G. C. Ramos, P. E. Davenport, A. R. Duncan, Y. S. Fraiman, L. C. Frazer, H. Healy, E. M. Herzberg, M. L. Keyes, K. T. Leeman, K. Leone, J. C. Levin, M. Lin, R. M. Raju, A. Sullivan, and Covid-Working Group Harvard Neonatal-Perinatal Fellowship. 2020. 'COVID19: neonatal-perinatal perspectives', J Perinatol.

Bischoff, A. L., N. V. Folsgaard, C. G. Carson, J. Stokholm, L. Pedersen, M. Holmberg, A. Bisgaard, S. Birch, T. F. Tsai, and H. Bisgaard. 2013. 'Altered response to $A(H 1 N 1)$ pnd09 vaccination in pregnant women: a single blinded randomized controlled trial', PLoS One, 8: e56700.

Bondt, A., Y. Rombouts, M. H. Selman, P. J. Hensbergen, K. R. Reiding, J. M. Hazes, R. J. Dolhain, and M. Wuhrer. 2014. 'Immunoglobulin G (IgG) Fab glycosylation analysis using a new mass spectrometric high-throughput profiling method reveals pregnancy-associated changes', Mol Cell Proteomics, 13: 3029-39.

Bondt, A., M. H. Selman, A. M. Deelder, J. M. Hazes, S. P. Willemsen, M. Wuhrer, and R. J. Dolhain. 2013. 'Association between galactosylation of immunoglobulin $G$ and improvement of rheumatoid arthritis during pregnancy is independent of sialylation', J Proteome Res, 12: 4522-31.

Bondt, A., M. Wuhrer, T. M. Kuijper, J. M. Hazes, and R. J. Dolhain. 2016. 'Fab glycosylation of immunoglobulin $\mathrm{G}$ does not associate with improvement of rheumatoid arthritis during pregnancy', Arthritis Res Ther, 18: 274.

Brown, E. P., A. F. Licht, A. S. Dugast, I. Choi, C. Bailey-Kellogg, G. Alter, and M. E. Ackerman. 2012. 'High-throughput, multiplexed IgG subclassing of antigen-specific antibodies from clinical samples', J Immunol Methods, 386: 117-23.

Bruckner, C., C. Lehmann, D. Dudziak, and F. Nimmerjahn. 2017. 'Sweet SIGNs: IgG glycosylation leads the way in IVIG-mediated resolution of inflammation', Int Immunol, 29: 499-509. 
Bruhns, P., B. lannascoli, P. England, D. A. Mancardi, N. Fernandez, S. Jorieux, and M. Daeron. 2009. 'Specificity and affinity of human Fcgamma receptors and their polymorphic variants for human IgG subclasses', Blood, 113: 3716-25.

Einarsdottir, H. K., M. H. Selman, R. Kapur, S. Scherjon, C. A. Koeleman, A. M. Deelder, C. E. van der Schoot, G. Vidarsson, and M. Wuhrer. 2013. 'Comparison of the Fc glycosylation of fetal and maternal immunoglobulin G', Glycoconj J, 30: 147-57.

Faucette, Azure N., Benjamin L. Unger, Bernard Gonik, and Kang Chen. 2015. 'Maternal vaccination: moving the science forward', Human Reproduction Update, 21: 119-35.

Fiore, A. E., T. M. Uyeki, K. Broder, L. Finelli, G. L. Euler, J. A. Singleton, J. K. Iskander, P. M. Wortley, D. K. Shay, J. S. Bresee, N. J. Cox, Control Centers for Disease, and Prevention. 2010. 'Prevention and control of influenza with vaccines: recommendations of the Advisory Committee on Immunization Practices (ACIP), 2010', MMWR Recomm Rep, 59: 1-62.

Fischinger, S., J. K. Fallon, A. R. Michell, T. Broge, T. J. Suscovich, H. Streeck, and G. Alter. 2019. 'A high-throughput, bead-based, antigen-specific assay to assess the ability of antibodies to induce complement activation', J Immunol Methods, 473: 112630.

Forsyth, K., S. Plotkin, T. Tan, and C. H. Wirsing von Konig. 2015. 'Strategies to decrease pertussis transmission to infants', Pediatrics, 135: e1475-82.

Grohskopf, L. A., E. Alyanak, K. R. Broder, L. H. Blanton, A. M. Fry, D. B. Jernigan, and R. L. Atmar. 2020. 'Prevention and Control of Seasonal Influenza with Vaccines: Recommendations of the Advisory Committee on Immunization Practices - United States, 2020-21 Influenza Season', MMWR Recomm Rep, 69: 1-24.

Gunn, B. M., and G. Alter. 2016. 'Modulating Antibody Functionality in Infectious Disease and Vaccination', Trends Mol Med, 22: 969-82.

Hjelholt, A., G. Christiansen, U. S. Sorensen, and S. Birkelund. 2013. 'IgG subclass profiles in normal human sera of antibodies specific to five kinds of microbial antigens', Pathog Dis, 67: 206-13.

Huygen, K., R. N. Cabore, K. Maertens, P. Van Damme, and E. Leuridan. 2015. 'Humoral and cell mediated immune responses to a pertussis containing vaccine in pregnant and nonpregnant women', Vaccine, 33: 4117-23.

Jacobsen, H., M. Rajendran, A. Choi, H. Sjursen, K. A. Brokstad, R. J. Cox, P. Palese, F. Krammer, and R. Nachbagauer. 2017. 'Influenza Virus Hemagglutinin Stalk-Specific Antibodies in Human Serum are a Surrogate Marker for In Vivo Protection in a Serum Transfer Mouse Challenge Model', MBio, 8.

Jamieson, D. J., M. A. Honein, S. A. Rasmussen, J. L. Williams, D. L. Swerdlow, M. S. Biggerstaff, S. Lindstrom, J. K. Louie, C. M. Christ, S. R. Bohm, V. P. Fonseca, K. A. Ritger, D. J. Kuhles, P. Eggers, H. Bruce, H. A. Davidson, E. Lutterloh, M. L. Harris, C. Burke, N. Cocoros, L. Finelli, K. F. MacFarlane, B. Shu, S. J. Olsen, and A. Pregnancy Working Group Novel Influenza. 2009. 'H1N1 2009 influenza virus infection during pregnancy in the USA', Lancet, 374: 451-8. 
Jansen, B. C., A. Bondt, K. R. Reiding, E. Lonardi, C. J. de Jong, D. Falck, G. S. Kammeijer, R. J. Dolhain, Y. Rombouts, and M. Wuhrer. 2016. 'Pregnancy-associated serum N-glycome changes studied by highthroughput MALDI-TOF-MS', Sci Rep, 6: 23296.

Jennewein, M. F., I. Goldfarb, S. Dolatshahi, C. Cosgrove, F. J. Noelette, M. Krykbaeva, J. Das, A. Sarkar, M. J. Gorman, S. Fischinger, C. M. Boudreau, J. Brown, J. H. Cooperrider, J. Aneja, T. J. Suscovich, B. S. Graham, G. M. Lauer, T. Goetghebuer, A. Marchant, D. Lauffenburger, A. Y. Kim, L. E. Riley, and G. Alter. 2019. 'Fc Glycan-Mediated Regulation of Placental Antibody Transfer', Cell.

Karsten, C. B., N. Mehta, S. A. Shin, T. J. Diefenbach, M. D. Slein, W. Karpinski, E. B. Irvine, T. Broge, T. J. Suscovich, and G. Alter. 2019. 'A versatile high-throughput assay to characterize antibodymediated neutrophil phagocytosis', J Immunol Methods, 471: 46-56.

Kosikova, M., L. Li, P. Radvak, Z. Ye, X. F. Wan, and H. Xie. 2018. 'Imprinting of Repeated Influenza A/H3 Exposures on Antibody Quantity and Antibody Quality: Implications for Seasonal Vaccine Strain Selection and Vaccine Performance', Clin Infect Dis, 67: 1523-32.

Kourtis, A. P., J. S. Read, and D. J. Jamieson. 2014. 'Pregnancy and infection', N Engl J Med, 370: 2211-8.

Kraus, T. A., S. M. Engel, R. S. Sperling, L. Kellerman, Y. Lo, S. Wallenstein, M. M. Escribese, J. L. Garrido, T. Singh, M. Loubeau, and T. M. Moran. 2012. 'Characterizing the pregnancy immune phenotype: results of the viral immunity and pregnancy (VIP) study', J Clin Immunol, 32: 300-11.

Kraus, T. A., R. S. Sperling, S. M. Engel, Y. Lo, L. Kellerman, T. Singh, M. Loubeau, Y. Ge, J. L. Garrido, M. Rodriguez-Garcia, and T. M. Moran. 2010. 'Peripheral blood cytokine profiling during pregnancy and post-partum periods', Am J Reprod Immunol, 64: 411-26.

Louie, J. K., M. Acosta, D. J. Jamieson, M. A. Honein, and Group California Pandemic Working. 2010. 'Severe 2009 H1N1 influenza in pregnant and postpartum women in California', N Engl J Med, 362: 2735.

Martinez, D. R., Y. Fong, S. H. Li, F. Yang, M. F. Jennewein, J. A. Weiner, E. A. Harrell, J. F. Mangold, R. Goswami, G. R. Seage, 3rd, G. Alter, M. E. Ackerman, X. Peng, G. G. Fouda, and S. R. Permar. 2019. 'Fc Characteristics Mediate Selective Placental Transfer of IgG in HIV-Infected Women', Cell.

Memoli, M. J., H. Harvey, D. M. Morens, and J. K. Taubenberger. 2013. 'Influenza in pregnancy', Influenza Other Respir Viruses, 7: 1033-9.

Monath, T. P., and A. Nasidi. 1993. 'Should yellow fever vaccine be included in the expanded program of immunization in Africa? A cost-effectiveness analysis for Nigeria', Am J Trop Med Hyg, 48: 274-99.

Mor, G., and I. Cardenas. 2010. 'The immune system in pregnancy: a unique complexity', Am J Reprod Immunol, 63: 425-33.

Munoz, F. M., S. M. Patel, L. A. Jackson, G. K. Swamy, K. M. Edwards, S. E. Frey, C. R. Petrie, E. A. Sendra, and W. A. Keitel. 2020. 'Safety and immunogenicity of three seasonal inactivated influenza vaccines among pregnant women and antibody persistence in their infants', Vaccine, 38: 5355-63. 
Ohfuji, S., M. Deguchi, D. Tachibana, M. Koyama, T. Takagi, T. Yoshioka, A. Urae, K. Ito, T. Kase, A. Maeda, K. Kondo, W. Fukushima, Y. Hirota, and Group Osaka Pregnant Women Influenza Study. 2018. 'Protective Effect of Maternal Influenza Vaccination on Influenza in Their Infants: A Prospective Cohort Study', J Infect Dis, 217: 878-86.

Pagan, J. D., M. Kitaoka, and R. M. Anthony. 2018. 'Engineered Sialylation of Pathogenic Antibodies In Vivo Attenuates Autoimmune Disease', Cell, 172: 564-77 e13.

Pica, N., R. Hai, F. Krammer, T. T. Wang, J. Maamary, D. Eggink, G. S. Tan, J. C. Krause, T. Moran, C. R. Stein, D. Banach, J. Wrammert, R. B. Belshe, A. Garcia-Sastre, and P. Palese. 2012. 'Hemagglutinin stalk antibodies elicited by the 2009 pandemic influenza virus as a mechanism for the extinction of seasonal H1N1 viruses', Proc Natl Acad Sci U S A, 109: 2573-8.

Radvak, P., M. Kosikova, Y. C. Kuo, X. Li, R. Garner, F. Schmeisser, I. Kosik, Z. Ye, J. P. Weir, J. W. Yewdell, and H. Xie. 2021. 'Highly pathogenic avian influenza A/Guangdong/17SF003/2016 is immunogenic and induces cross-protection against antigenically divergent H7N9 viruses', NPJ Vaccines, 6: 30 .

Regan, A. K., N. de Klerk, H. C. Moore, S. B. Omer, G. Shellam, and P. V. Effler. 2016. 'Effect of Maternal Influenza Vaccination on Hospitalization for Respiratory Infections in Newborns: A Retrospective Cohort Study', Pediatr Infect Dis J, 35: 1097-103.

Rolle, L., M. Memarzadeh Tehran, A. Morell-Garcia, Y. Raeva, A. Schumacher, R. Hartig, S. D. Costa, F. Jensen, and A. C. Zenclussen. 2013. 'Cutting edge: IL-10-producing regulatory B cells in early human pregnancy', Am J Reprod Immunol, 70: 448-53.

Ruhaak, L. R., H. W. Uh, A. M. Deelder, R. E. Dolhain, and M. Wuhrer. 2014. 'Total plasma N-glycome changes during pregnancy', J Proteome Res, 13: 1657-68.

Safety, Global Advisory Committee on Vaccine. 2014. "Safety and immunization during pregnancy." In.: World Health Organizatioj.

Saso, A., and B. Kampmann. 2020. 'Maternal Immunization: Nature Meets Nurture', Front Microbiol, 11: 1499.

Sautto, G. A., G. A. Kirchenbaum, and T. M. Ross. 2018. 'Towards a universal influenza vaccine: different approaches for one goal', Virol J, 15: 17.

Schlaudecker, E. P., L. Ambroggio, M. M. McNeal, F. D. Finkelman, and S. S. Way. 2018. 'Declining responsiveness to influenza vaccination with progression of human pregnancy', Vaccine, 36: 4734-41.

Schlaudecker, E. P., M. M. McNeal, C. N. Dodd, J. B. Ranz, and M. C. Steinhoff. 2012. 'Pregnancy modifies the antibody response to trivalent influenza immunization', J Infect Dis, 206: 1670-3.

Schumacher, A., S. D. Costa, and A. C. Zenclussen. 2014. 'Endocrine factors modulating immune responses in pregnancy', Front Immunol, 5: 196.

Selman, M. H., S. E. de Jong, D. Soonawala, F. P. Kroon, A. A. Adegnika, A. M. Deelder, C. H. Hokke, M. Yazdanbakhsh, and M. Wuhrer. 2012. 'Changes in antigen-specific IgG1 Fc N-glycosylation upon influenza and tetanus vaccination', Mol Cell Proteomics, 11: M111.014563. 
Siston, A. M., S. A. Rasmussen, M. A. Honein, A. M. Fry, K. Seib, W. M. Callaghan, J. Louie, T. J. Doyle, M. Crockett, R. Lynfield, Z. Moore, C. Wiedeman, M. Anand, L. Tabony, C. F. Nielsen, K. Waller, S. Page, J. M. Thompson, C. Avery, C. B. Springs, T. Jones, J. L. Williams, K. Newsome, L. Finelli, D. J. Jamieson, and H. N. Influenza in Pregnancy Working Group Pandemic. 2010. 'Pandemic 2009 influenza A(H1N1) virus illness among pregnant women in the United States', JAMA, 303: 1517-25.

Sperling, R. S., S. M. Engel, S. Wallenstein, T. A. Kraus, J. Garrido, T. Singh, L. Kellerman, and T. M. Moran. 2012. 'Immunogenicity of trivalent inactivated influenza vaccination received during pregnancy or postpartum', Obstet Gynecol, 119: 631-9.

Stevens, J., A. L. Corper, C. F. Basler, J. K. Taubenberger, P. Palese, and I. A. Wilson. 2004. 'Structure of the uncleaved human $\mathrm{H} 1$ hemagglutinin from the extinct 1918 influenza virus', Science, 303: 186670.

Stewart, Ross, Scott A. Hammond, Michael Oberst, and Robert W. Wilkinson. 2014. 'The role of Fc gamma receptors in the activity of immunomodulatory antibodies for cancer', Journal for ImmunoTherapy of Cancer, 2: 29.

Thompson, M. G., J. C. Kwong, A. K. Regan, M. A. Katz, S. J. Drews, E. Azziz-Baumgartner, N. P. Klein, H. Chung, P. V. Effler, B. S. Feldman, K. Simmonds, B. E. Wyant, F. S. Dawood, M. L. Jackson, D. B. Fell, A. Levy, N. Barda, L. W. Svenson, R. V. Fink, S. W. Ball, A. Naleway, and Prevent Workgroup. 2019. 'Influenza Vaccine Effectiveness in Preventing Influenza-associated Hospitalizations During Pregnancy: A Multi-country Retrospective Test Negative Design Study, 2010-2016', Clin Infect Dis, 68: 1444-53.

van de Bovenkamp, F. S., L. Hafkenscheid, T. Rispens, and Y. Rombouts. 2016. 'The Emerging Importance of IgG Fab Glycosylation in Immunity', J Immunol, 196: 1435-41.

van de Geijn, F. E., M. Wuhrer, M. H. Selman, S. P. Willemsen, Y. A. de Man, A. M. Deelder, J. M. Hazes, and R. J. Dolhain. 2009. 'Immunoglobulin G galactosylation and sialylation are associated with pregnancy-induced improvement of rheumatoid arthritis and the postpartum flare: results from a large prospective cohort study', Arthritis Res Ther, 11: R193.

Vojtek, I., I. Dieussaert, T. M. Doherty, V. Franck, L. Hanssens, J. Miller, R. Bekkat-Berkani, W. Kandeil, D. Prado-Cohrs, and A. Vyse. 2018. 'Maternal immunization: where are we now and how to move forward?', Ann Med, 50: 193-208.

Watanabe, M., Y. Iwatani, T. Kaneda, Y. Hidaka, N. Mitsuda, Y. Morimoto, and N. Amino. 1997. 'Changes in T, B, and NK lymphocyte subsets during and after normal pregnancy', Am.J.Reprod.Immunol., 37: 368-77.

WHO. 'WHO recommendations on the composition of influenza virus vaccines', Accessed March 23rd. https://www.who.int/influenza/vaccines/virus/recommendations/en/.

Wu, N. C., and I. A. Wilson. 2017. 'A Perspective on the Structural and Functional Constraints for Immune Evasion: Insights from Influenza Virus', J Mol Biol, 429: 2694-709.

Xie, H., X. Jing, X. Li, Z. Lin, E. Plant, O. Zoueva, H. Yang, and Z. Ye. 2011. 'Immunogenicity and crossreactivity of 2009-2010 inactivated seasonal influenza vaccine in US adults and elderly', PLoS One, 6: e16650. 
Xie, H., X. F. Wan, Z. Ye, E. P. Plant, Y. Zhao, Y. Xu, X. Li, C. Finch, N. Zhao, T. Kawano, O. Zoueva, M. J. Chiang, X. Jing, Z. Lin, A. Zhang, and Y. Zhu. 2015. 'H3N2 Mismatch of 2014-15 Northern Hemisphere Influenza Vaccines and Head-to-head Comparison between Human and Ferret Antisera derived Antigenic Maps', Sci Rep, 5: 15279.

Yee, J., W. Kim, J. M. Han, H. Y. Yoon, N. Lee, K. E. Lee, and H. S. Gwak. 2020. 'Clinical manifestations and perinatal outcomes of pregnant women with COVID-19: a systematic review and meta-analysis', Sci Rep, 10: 18126.

Zambrano, L. D., S. Ellington, P. Strid, R. R. Galang, T. Oduyebo, V. T. Tong, K. R. Woodworth, J. F. Nahabedian, 3rd, E. Azziz-Baumgartner, S. M. Gilboa, D. Meaney-Delman, Cdc Covid- Response Pregnancy, and Team Infant Linked Outcomes. 2020. 'Update: Characteristics of Symptomatic Women of Reproductive Age with Laboratory-Confirmed SARS-CoV-2 Infection by Pregnancy Status - United States, January 22-October 3, 2020', MMWR Morb Mortal Wkly Rep, 69: 1641-47. 

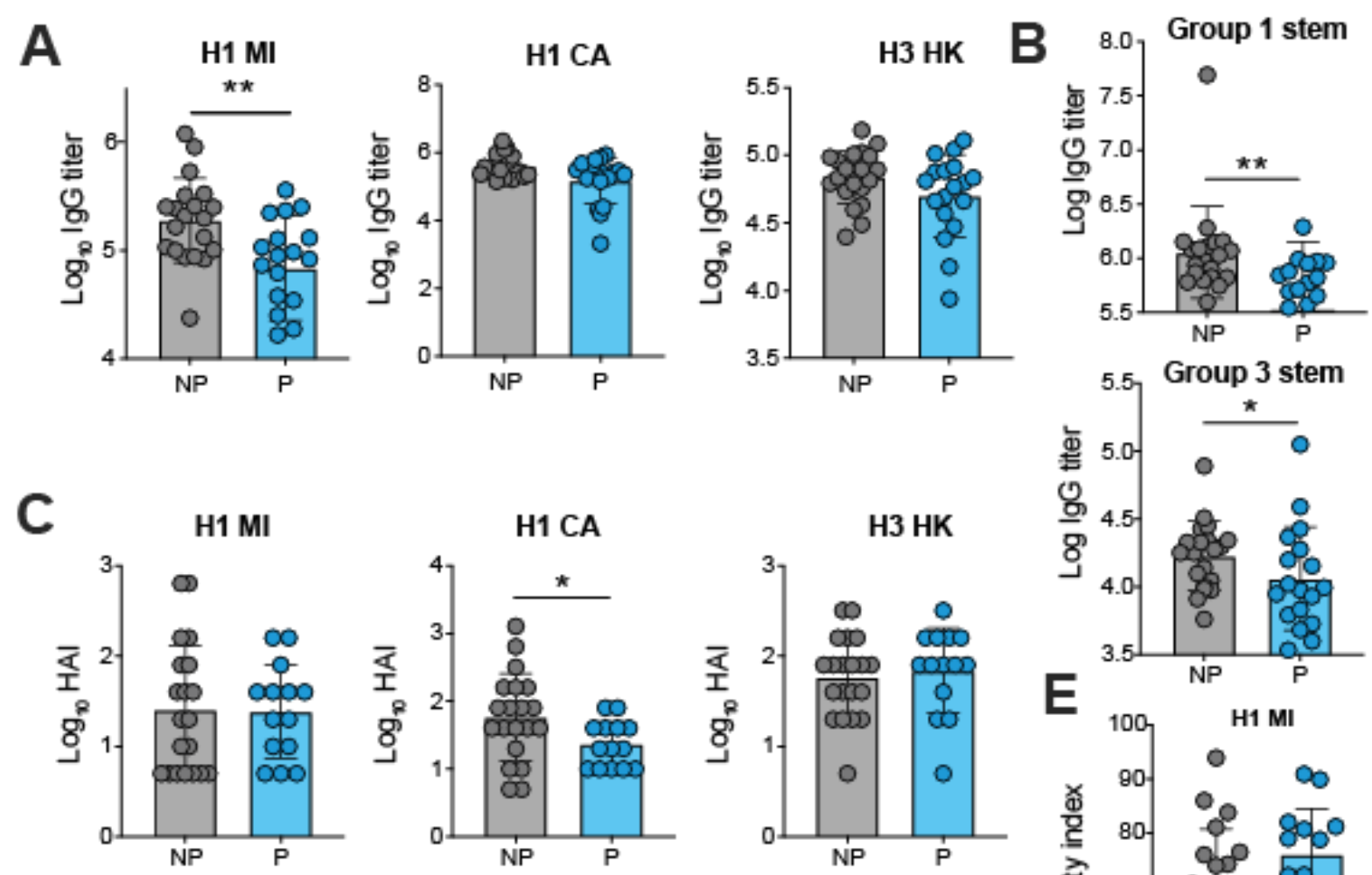

D

H1 MI

H1 CA
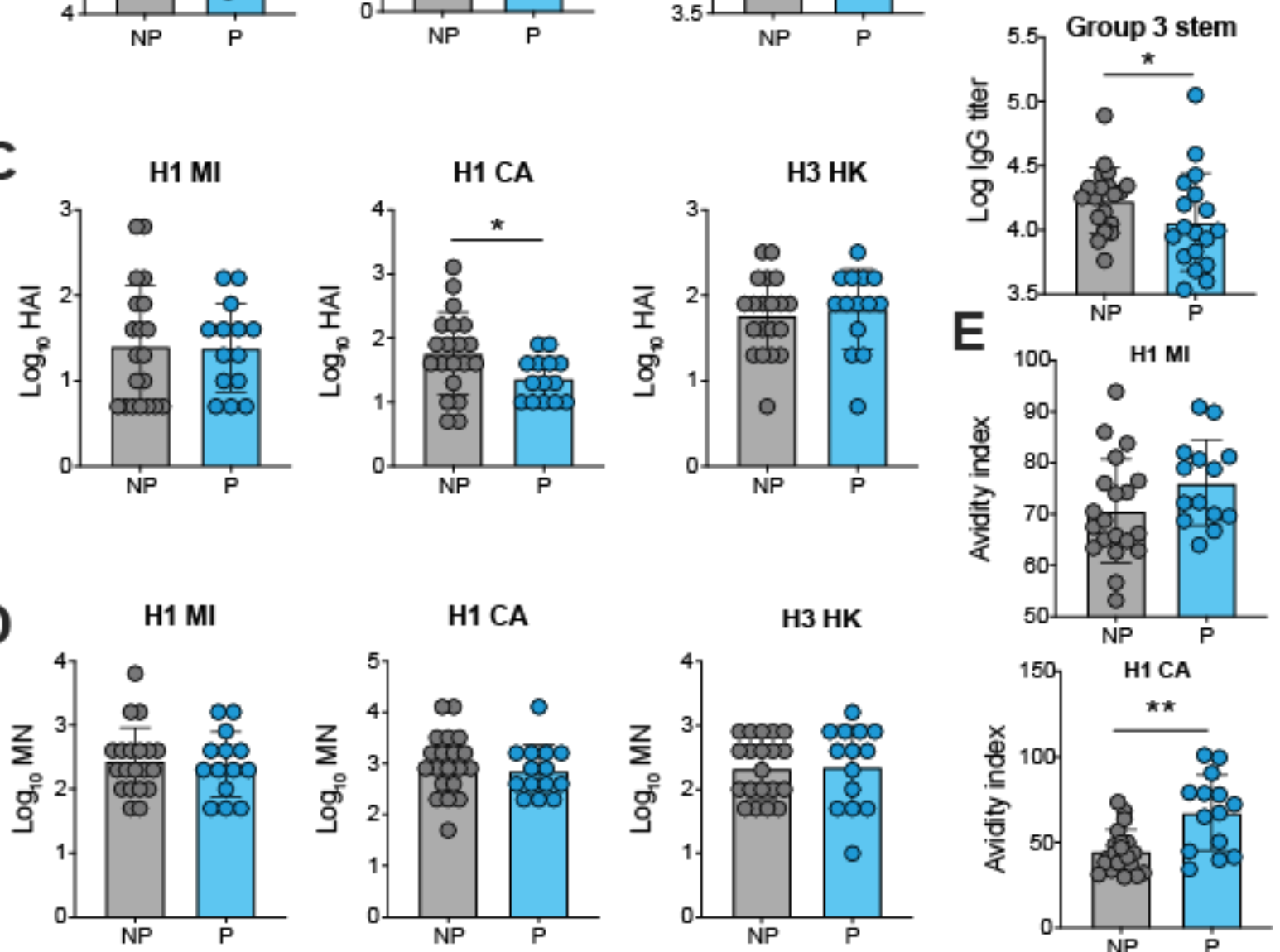

Figure 1: Pregnancy associated differences in influenza-specific antibody titer. HA-specific lgG (A), group 1- and group 3-stem specific IgG (B), hemagglutination inhibition (HAI) (C), microneutralization (MN) (D) and HA-specific lgG avidity (E) in pregnant and non-pregnant women. Data represent log transformed individual datapoints and geometric mean titers (bars). ELISA, HAI and MN titers were compared using one-tailed Mann-Whitney test. Avidity was evaluated using a two-tailed Mann-Whitney test ${ }^{*} p<0.05,{ }^{* *} p<0.01$. 
A
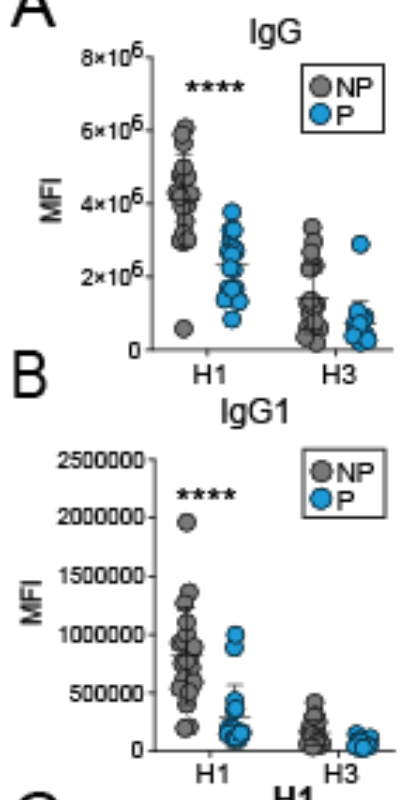

C
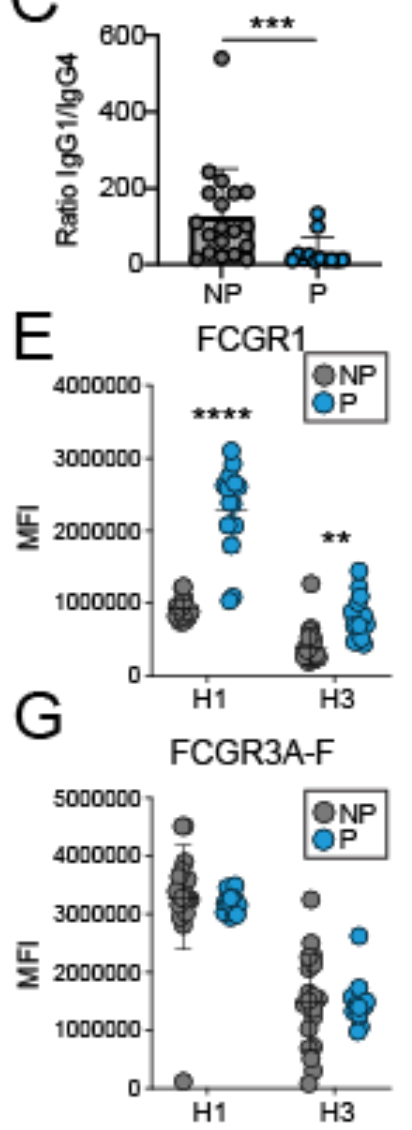
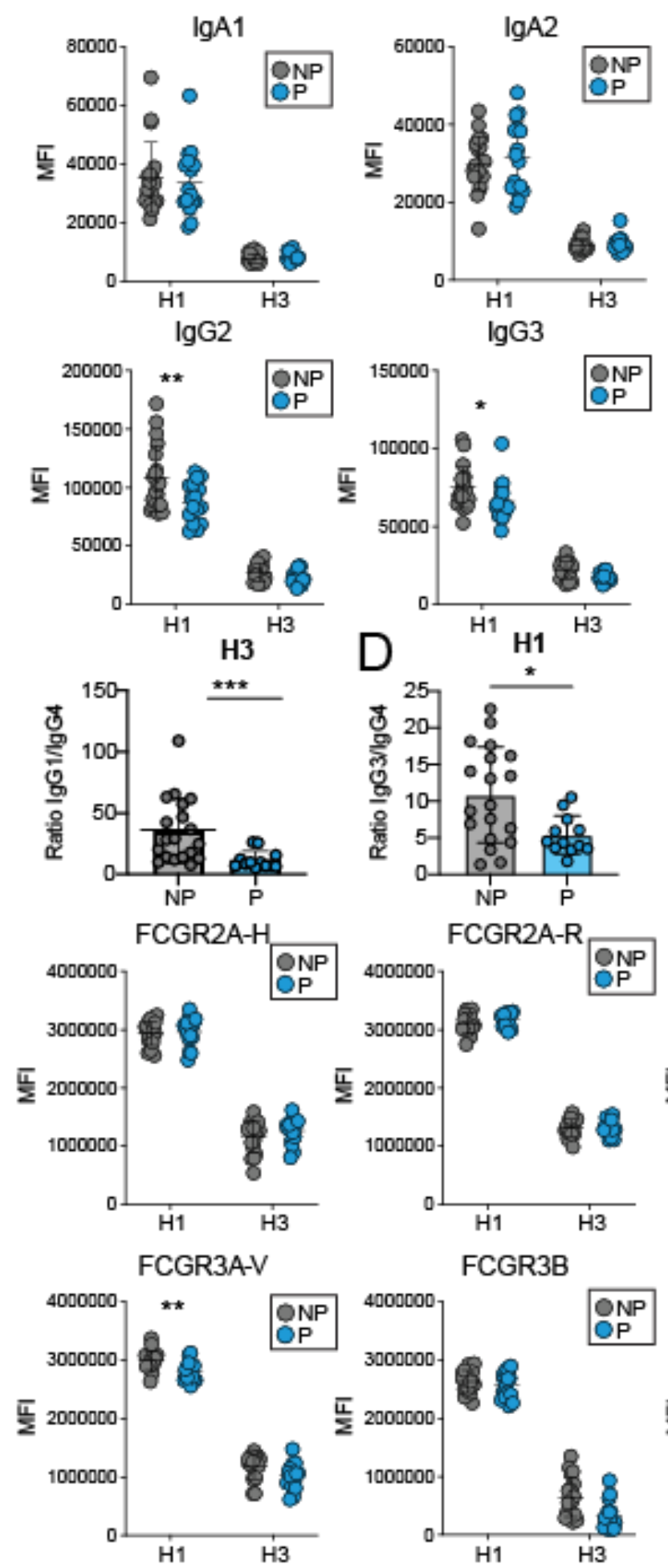

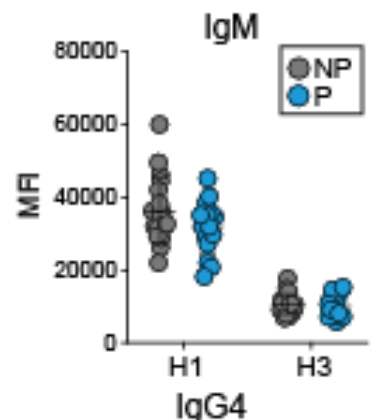

801
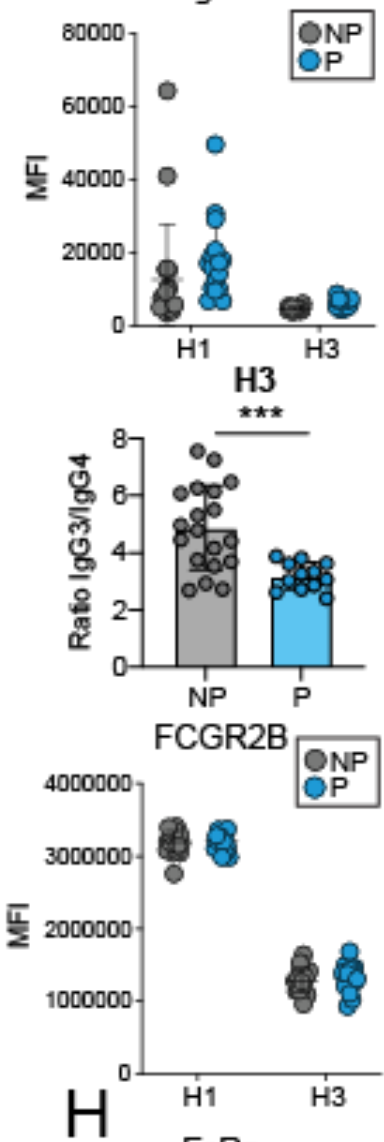

802

803

804 
805 Figure 2: Pregnancy influences HA-specific IgG subclass and Fc receptor binding.

$806 \lg$ and IgG subclass levels and the Fc receptor binding were assessed by luminex. A-B. Dot plots depict

807 the differences between isotype and subclass levels for non-pregnant (NP, grey circles) and pregnant (P, 808 blue circles) women. A. Isotypes. B. IgG subclasses. C-D. IgG1/lgG4 (C) IgG3/lgG4 ratios (D). E-H. 809 Class 1 Fc receptor. D. Class 2 Fc receptors. E. Class 3 Fc receptors. F. Neonatal Fc receptor. Statistics 810 evaluated as Mann-Whitney test and stars represent differences between $\mathrm{H} 1$ and H3 response. ${ }^{*} \mathrm{p}<0.05$, $811{ }^{* *} p<0.01,{ }^{* * *} p<0.001,{ }^{* * * *} p<0.0001$. 
A Fc Bulk

$\mathrm{G} 0 * *$


B Fab Bulk

GO

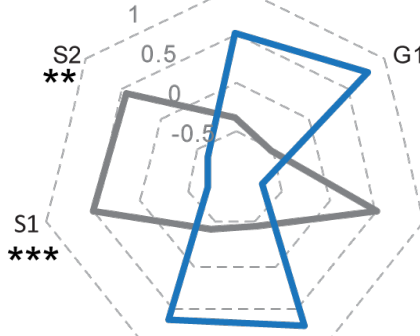

** $\quad * * * *$

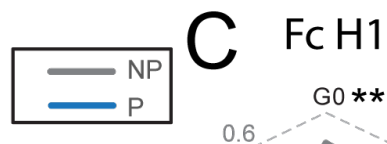

G2 $\mathrm{S} 1$

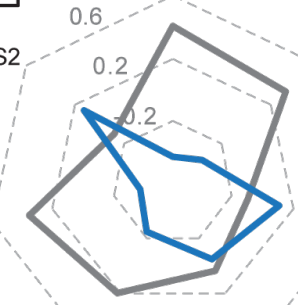

B

$D$
G2S1
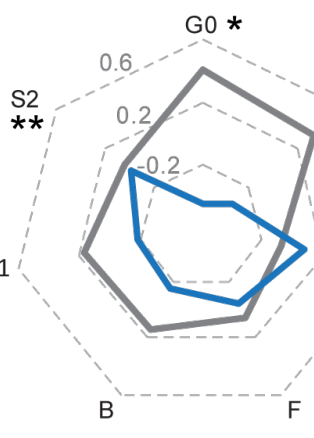

E Fab H1

G0

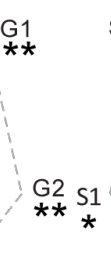

B

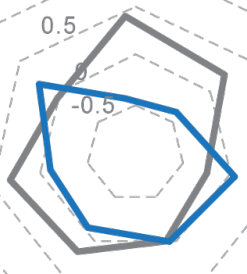

F FabH3

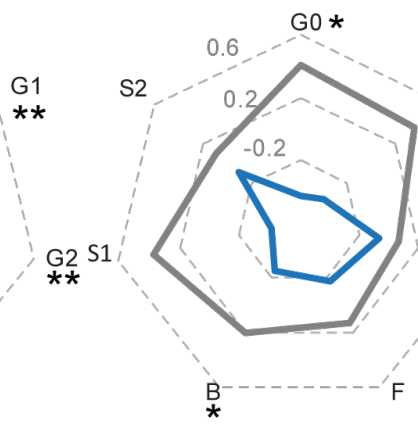

W

Fab-G1

* Fab-G2

Fab-F

Fab-B

G2 Fab-S1

Fab-S2

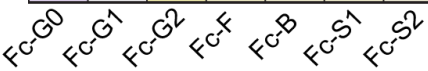

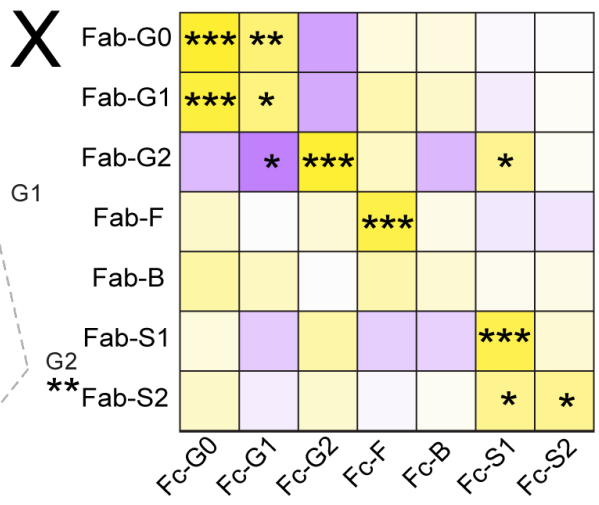

GGalactosylation ${ }_{100}$ Fucosylation $J$ Sialylation $\mathrm{K}$ Galactosylation $\square \mathrm{G} 2 \square \mathrm{G} 1 \square \mathrm{G} 0$

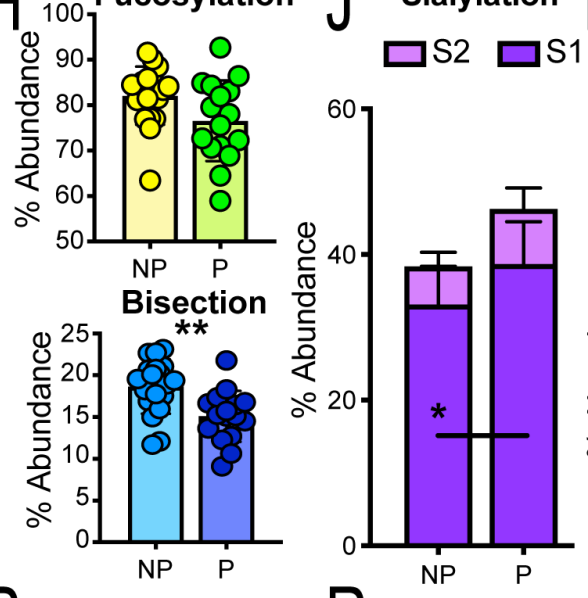

Galactosylation $P$
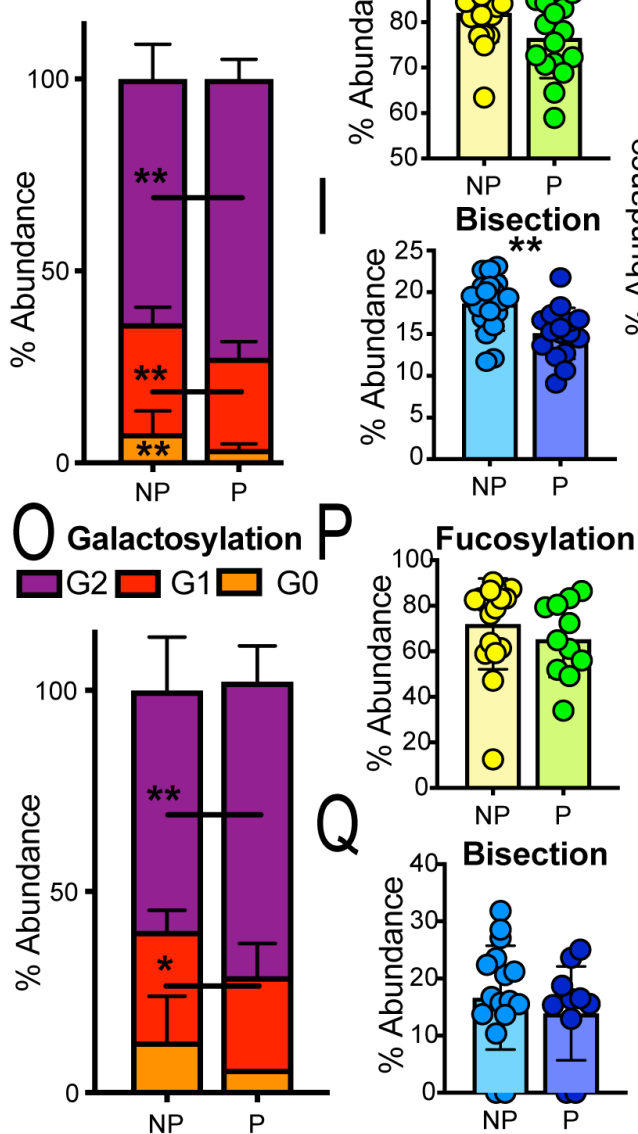

$\mathrm{R}$ Sialylation


$N$ Sialylation
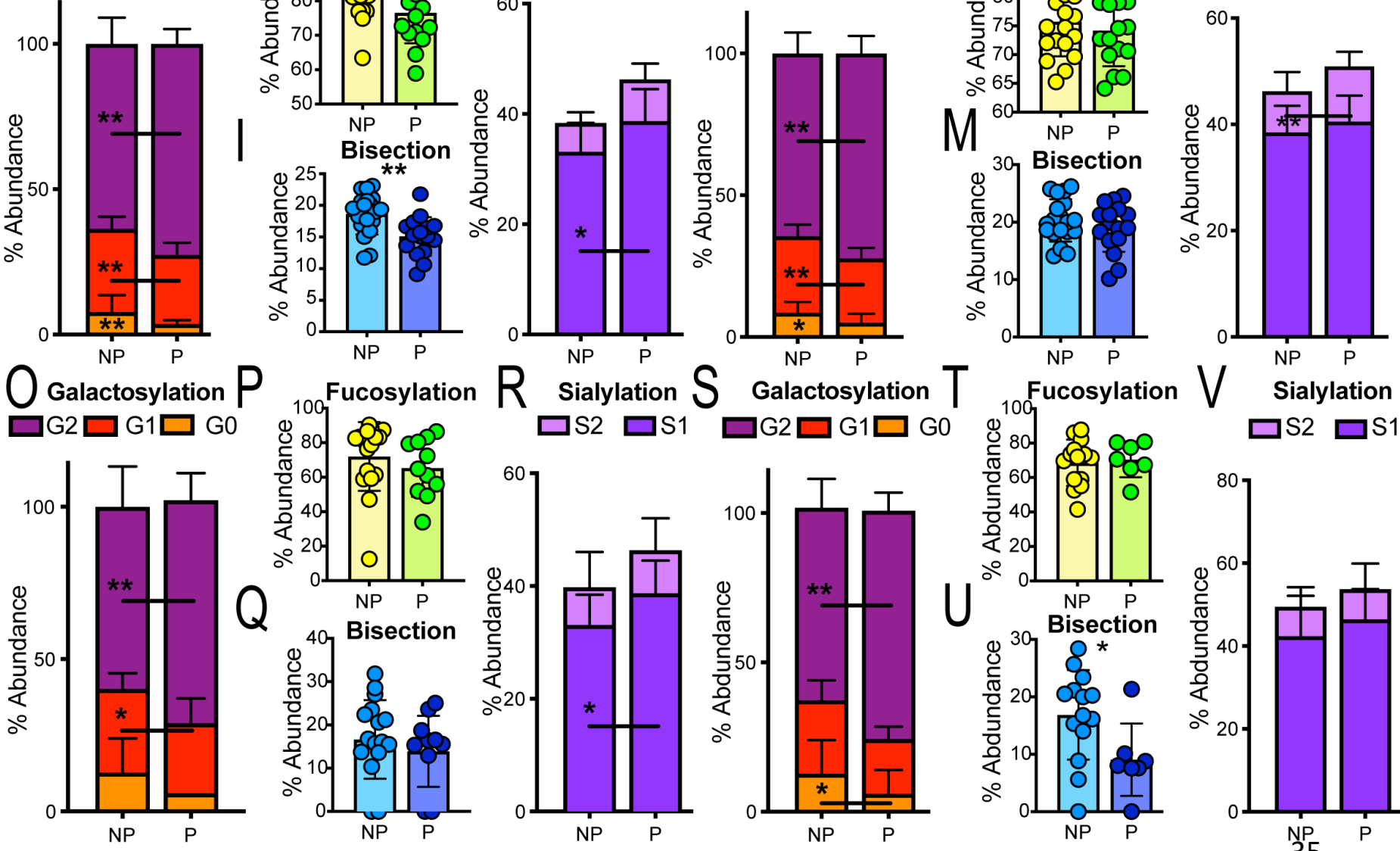

Sialylation

$\square S 2 \square S 1$

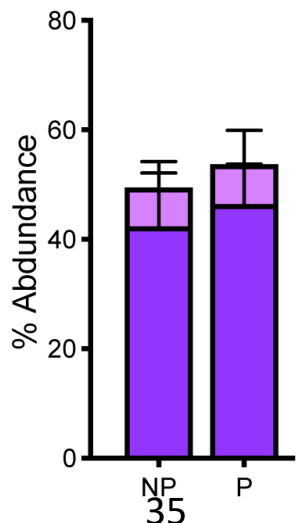




\section{Figure 3: Pregnancy alters antibody Fc and Fab glycosylation.}

816 Antibody glycosylation for HA-specific and bulk antibodies was analyzed by capillary electrophoresis.

817 A-R. Radar plots depicting different glycosylation profiles of bulk lgG Fc (A) and Fab (B), H1-specific 818 IgG Fc (C) and Fab (E) and H3-specific IgG Fc (D) and Fab (E) in non-pregnant and pregnant groups. $819 \mathrm{G}=$ galactose (0-2), F=fucose, B=Bisecting GlcNAc, S=sialic acid (1-2). G-V. These dot-line and 820 stacked-bar plots depict the differences in HA1-specific antibody Fc (G-J) and Fab (O-R) and HA3821 specific antibody Fc (K-N) and Fab (S-V) glycosylation between non-pregnant (NP) and pregnant (P) 822 women. (W, X) Heatmaps showing the spearman correlation between HA-specific Fab and FC 823 glycosylation for pregnant $(\mathbf{W})$ and non-pregnant $(\mathbf{X})$ women. Significance for radar plots and 824 glycosylation determined by Mann-Whitney test. ${ }^{*} p<0.05,{ }^{* *} p<0.01,{ }^{* * *} p<0.001,{ }^{* * *} p<0.0001$. 


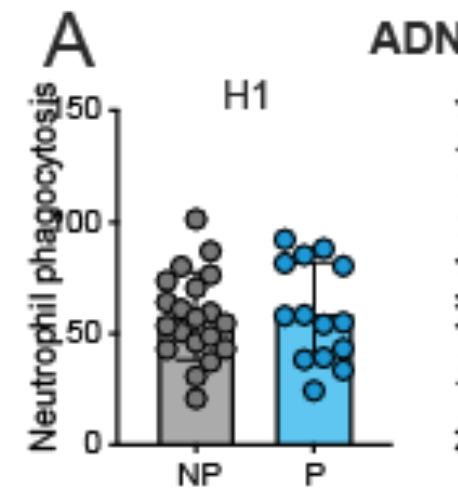

ADNP
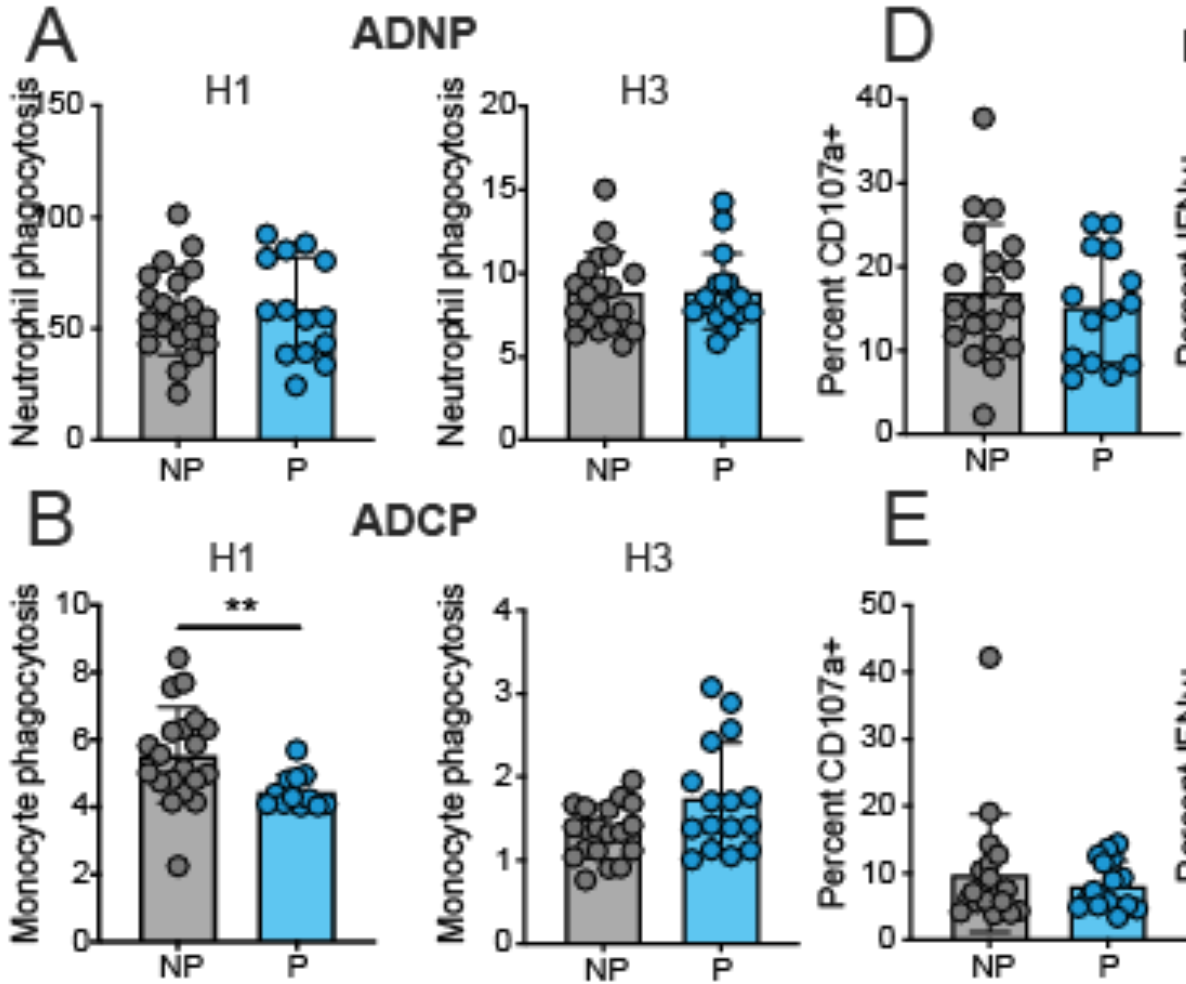

H1 NK Degranulation
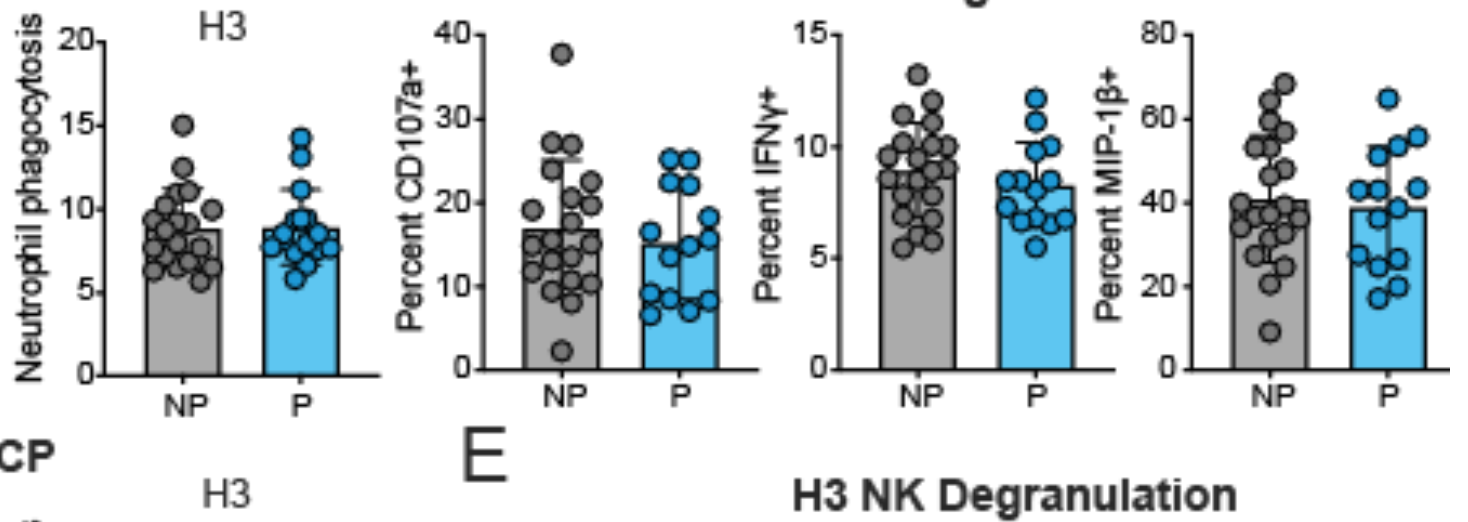

H3 NK Degranulation
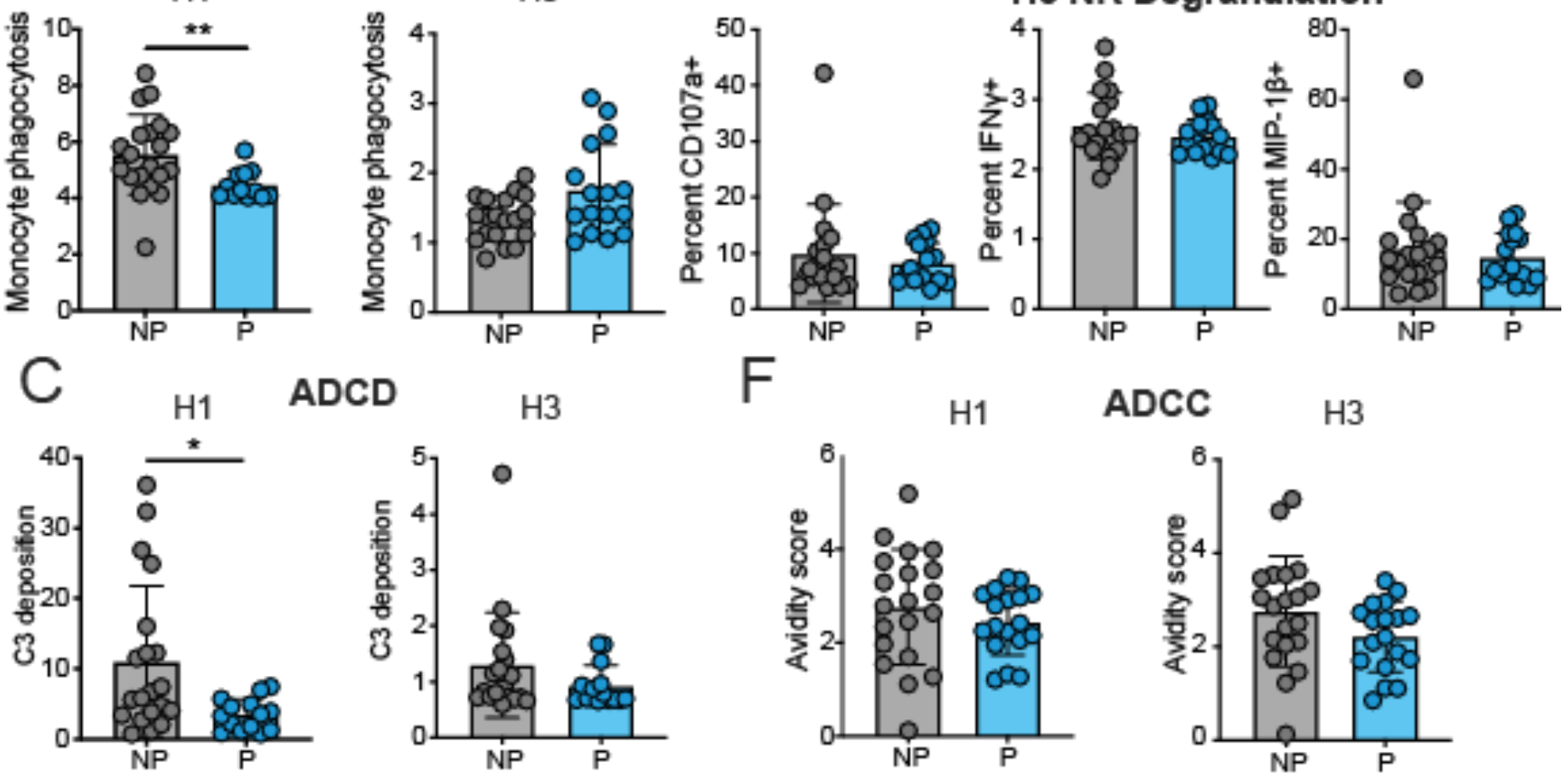

Figure 4: Antigen-specific non-neutralizing antibody functions are affected by pregnancy.

828 Influenza H1- and H3-specific antibody dependent neutrophil phagocytosis (ADNP) (A), monocyte

829 phagocytosis (ADCP) (B), complement deposition (ADCD) (C), NK degranulation measured as

830 CD107a, MIP-1 $\beta$ and IFNy (D, E) and cellular cytotoxicity (ADCC) (F). Groups were compared using 831 two-tailed Mann-Whitney Test. ${ }^{*} p<0.05,{ }^{* *} p<0.01$. 


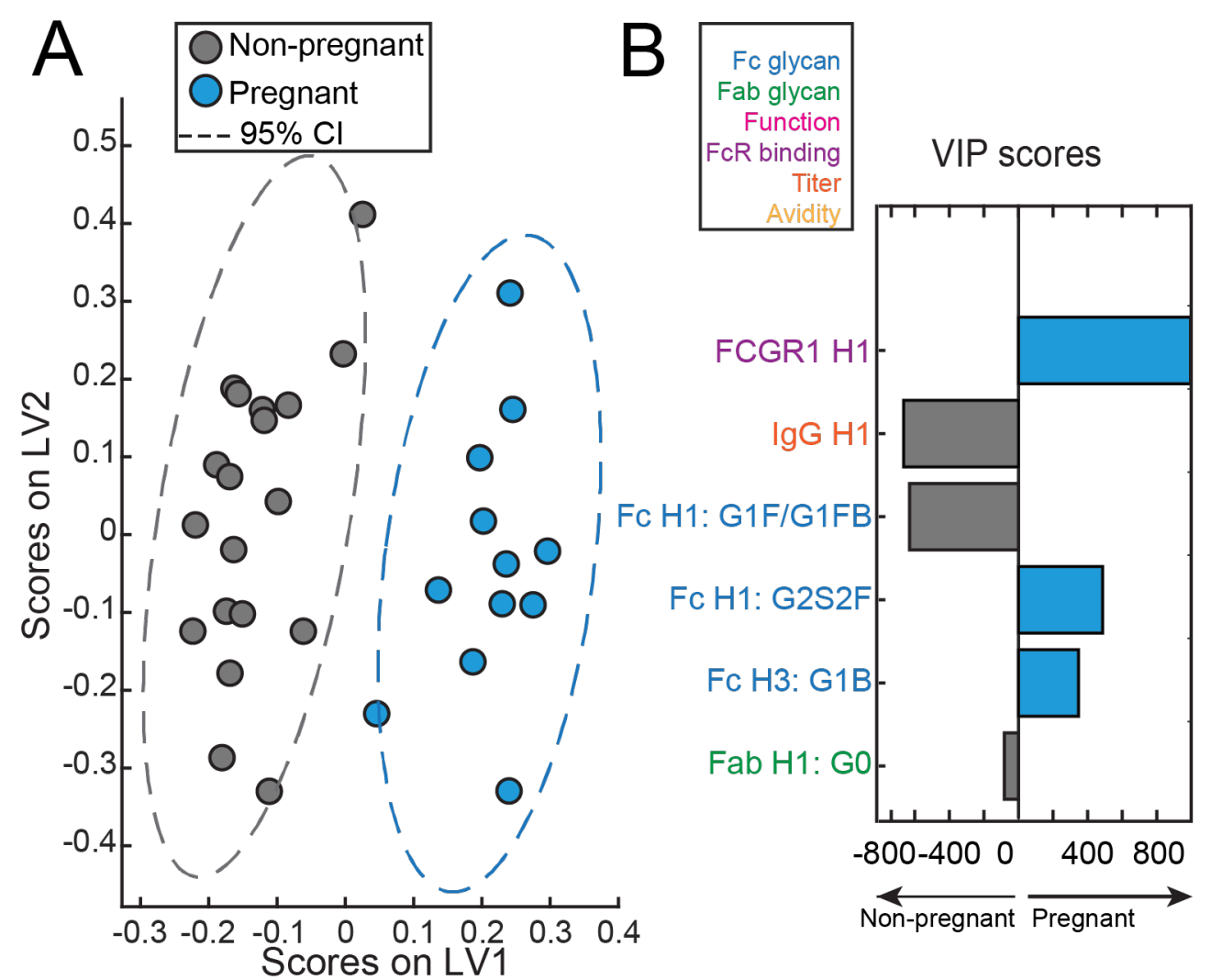

836 Figure 5: Multivariate signatures of antibodies produced during pregnancy.

837 Computational analysis was used to distinguish features enriched in pregnancy. A. LASSO Partial 838 Least Squares Discriminant Analysis (LASSO-PLSDA) of influenza-specific antibody features between 839 pregnant and non-pregnant women orthogonalized along latent variable 1 (LV1). LV1 explains $42.5 \%$ of 840 the variance along the $X$ axis while LV2 explained $17.1 \%$ of the variance. $B$. The Variable Importance in 841 Projection (VIP) scores for the PLSDA indicate the prime factors driving the differences between

842 pregnant and non-pregnant women. Factors pointing towards the left are enriched in non-pregnant

843 samples, while those pointing right are enriched in pregnant samples. Bar color and length corresponds 844 to relative importance. Antibody features are colored by category. 


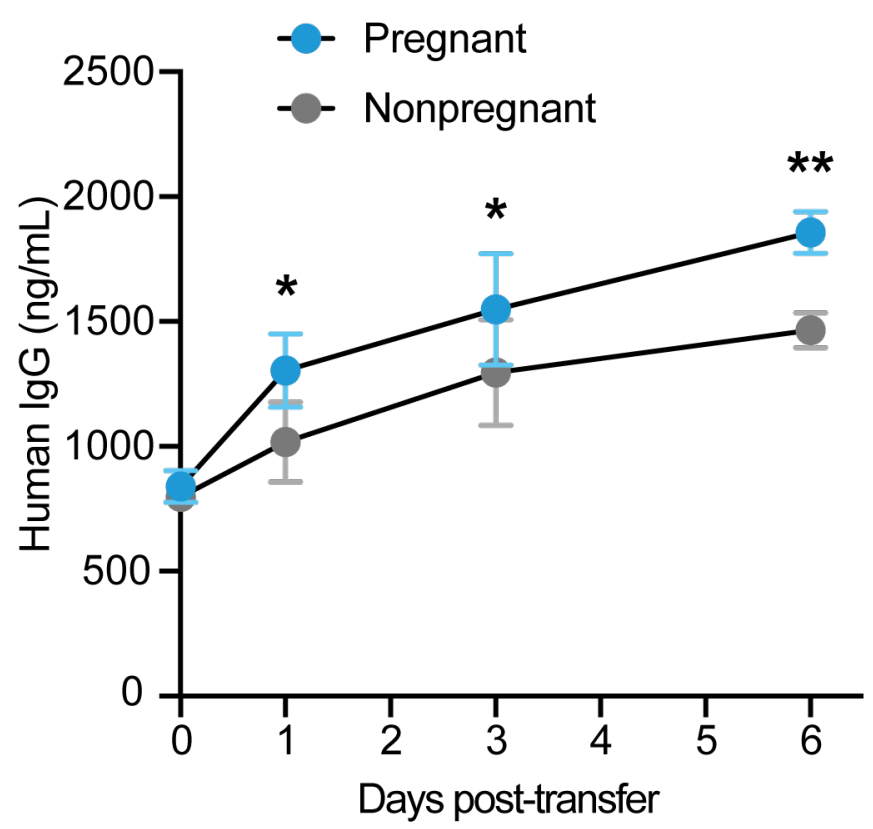

\section{Figure 6. In vivo antibody retention.}

849 The dot-line plot depicts the retention of lgG in mice injected with pooled plasma from pregnant (blue, 4 850 mice) or non-pregnant (grey, 5 mice) mice. Retention was measured over a 6-day period. Dots are at 851 mean with error bars at the standard deviation. Statistics evaluated using a two-way ANOVA. ${ }^{*} p<0.05$, $852{ }^{* *} \mathrm{p}<0.01$. 

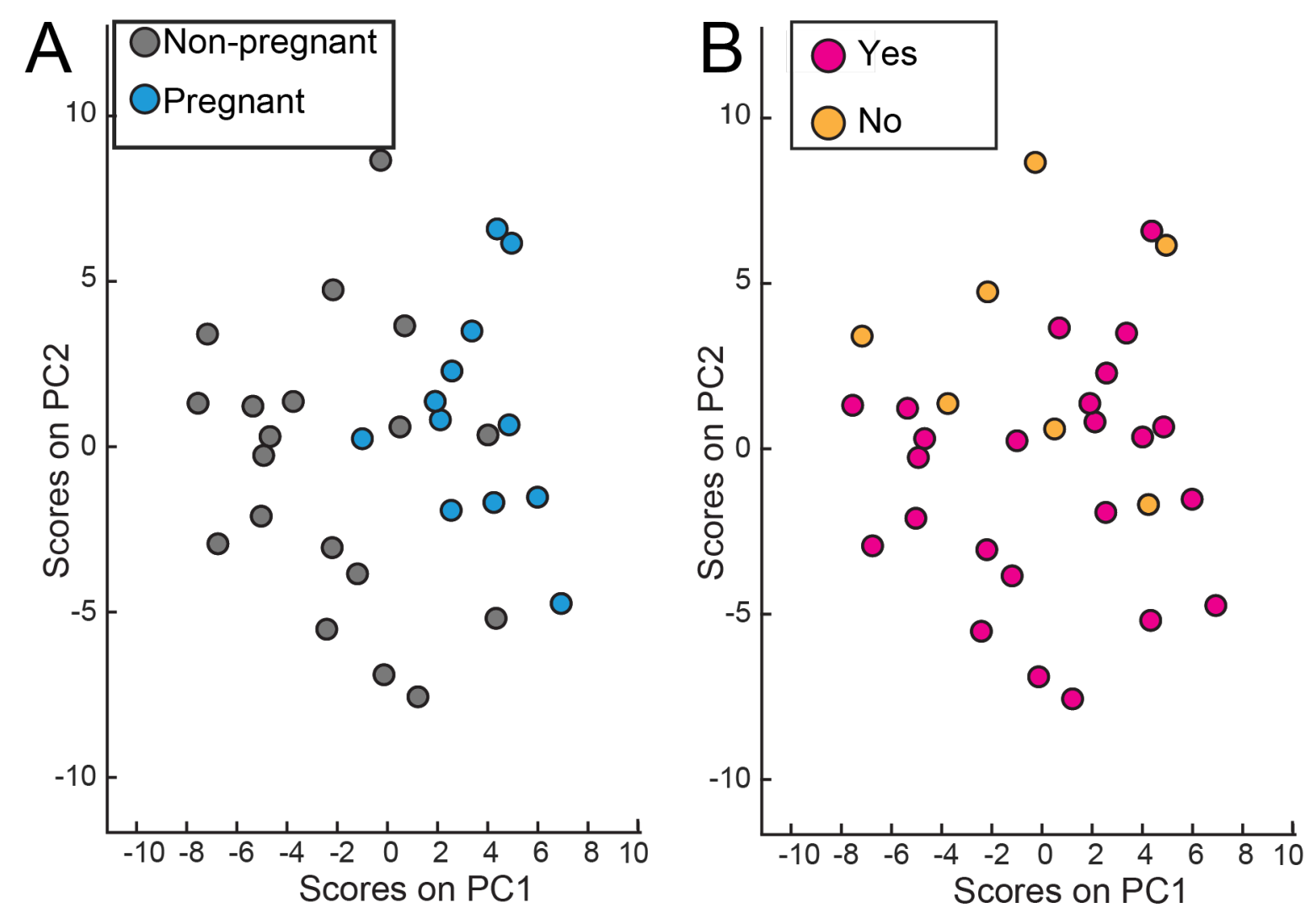

856 Supplemental Figure 1: Relationship of antibody profiles and vaccination.

857 PCA analysis was used to distinguish effects of vaccination. No differences were observed across the 858 antibody profiles for vaccination and un-vaccinated participants. Principle component 1 (PC1) contains $85912 \%$ of the variance and PC2 contains $10.5 \%$ of the variance. A. Dots colored to distinguish pregnant 860 (blue dots) and non-pregnant (grey dots) women. B. Dots colored to distinguish women who received 861 seasonal influenza vaccine (pink dots) and women without clinical record of vaccination (orange dots). 